\title{
Uncertainty and Sensitivity Analysis Applied to an In-Host Malaria Model with Multiple Vaccine Antigens
}

\author{
Titus Okello Orwa ${ }^{1} \cdot$ Rachel Waema Mbogo ${ }^{1} \cdot$ Livingstone Serwadda Luboobi $^{1}$
}

Published online: 24 May 2019

(c) Springer Nature India Private Limited 2019

\begin{abstract}
Results of uncertainty and sensitivity analysis have significant epidemiological importance in malaria control. In this paper, the efficient technique of latin hypercube sampling (LHS) and partial rank correlation coefficient is applied to an in-host Plasmodium falciparum malaria model. Sensitivity indices of the basic reproduction number are derived using the normalised forward approach. By a theoretical analysis, we show the existence and stability of the model steady states based on threshold value $R_{0}$. Our results show that the in-host model is sensitive to variations in the efficacy of malaria vaccines. The rates of parasite to cell invasions, the density of merozoites released per bursting infected erythrocyte and the proportions of merozoites that become gametocytes are highly significant in determining the severity of malaria infection. Moreover, a highly effective vaccine combination is critical for malaria disease elimination goal. This study further shows that the long term precise predictions of the concentrations of infected cells during malaria infection would be difficult until these key parameters are correctly determined. These results are vital in the on-going malaria vaccine development.
\end{abstract}

Keywords Sensitivity analysis · Hepatocytes $\cdot$ Erythrocytes $\cdot$ P. falciparum $\cdot$ Vaccine combination

\section{Introduction}

During disease model formulation, we are bound to make simplifications and assumptions on the model itself and on the parameters that represent the different transitions and interactions in the model. Owing to uncertainty on parameter values, it is important to correctly understand the possible effects of the such parameter values to the anticipated model output [21,52]. Uncertainty in the set of parameter values generates variability in the model's predictive capabilities. The lower the number of uncertain parameters in a model, the lower the significance of variability introduced into a model. On the other hand, a higher number of uncertain parameters result into a higher significance of variability introduced [21].

Titus Okello Orwa

torwa@strathmore.edu

1 Institute of Mathematical Sciences, Strathmore University, P.O Box 59857-00200, Nairobi, Kenya 
In-host malaria transmission models have become very complex. Unlike simple models, complex models may best be understood by numerical analysis [8]. Uncertainty and sensitivity analysis are instrumental in analyzing the dynamics of such structurally complex models that experiences high levels of uncertainty in the estimation of their input parameter values. The concept of uncertainty analysis enables us to evaluate the variability in the output [30]. Conversely, a sensitivity analysis helps us to discern important parameters in the dynamics of infection under study. There are three techniques of uncertainty and sensitivity analysis [30]. These include: (i) latin hypercube sampling/partial rank correlation coefficient (LHS/PRCC), (ii) response surface methodology, and (3) differential analysis. The more sophisticated and efficient approach of LHS/PRCC [9,30] that permits synchronous variation of all input variables is considered in this study.

LHS/PRCC sensitivity analysis is a synergy of Latin Hypercube Sampling [38] and the Partial Rank Correlation Coefficient [26]. It aims to identify and rank pivotal model parameters whose uncertainties contribute to prediction imprecision [21]. In this paper, uncertainty and sensitivity analysis is applied to an in-host malaria model subject to malaria vaccines. We endevour to identify influential parameters in determining malaria disease progression and control within the human host. See [2,50] for a rigorous description of the different malaria vaccines.

Malaria is a mosquito-borne infectious disease caused by Plasmodium parasite. The disease is typically transmitted through the bite of an infected mosquito vector [20]. The injected sporozoites traverse host dermis and enter the liver hepatocytes for further asexual development. Hepatocite-invading sporozoites, grow, divide mitotically and differentiate into liver merozoites [12]. The release of these merozoites into blood stream marks the beginning of the blood stage cycle. The merozoites invade susceptible erythrocytes to feed on hemoglobin. Like the liver stage, the merozoites also differentiate, causing infected erythrocytes to burst open, releasing more merozoites into blood stream. Higher densities of blood merozoites increases the severity of clinical malaria. The daughter merozoites quickly invade other red blood cells to renew the cycle or develop into male or female gametocytes [13]. This cycle is associated with malaria characteristics symptoms [17,69]. See [6,49] for a detailed discussion on stages of malaria infection. Although malaria is both preventable and curable, severe malaria may lead to seizures, coma and death [22].

Currently, there are more than 30 malaria vaccine candidates under development [68]. These vaccines are generally categorised into: (1) pre-erythrocytic vaccines (PEV), (2) blood stage vaccines (BSV) and (3) transmission blocking vaccines (TBV) [6]. RTS,S/AS01 (also known as Mosquirix) is the only malaria vaccine that has received a positive scientific opinion from the European Medicines Agency (EMA) [18]. This pre-erythrocytic vaccine has been evaluated in large phase 3 trial in sub-Saharan Africa. The vaccine showed a $45.7 \%(95 \%$ CI, 41.7-49.5) efficacy against all episodes of clinical $P$. falciparum malaria across all sites in the 5-17 month age category [54]. Despite positive assessment from EMA, the World Health Organization (WHO) recomments further evaluation of RTS,S/AS01 in a series of pilot implementations before it can be introduced on a wide scale [70]. Nonetheless, an efficacious malaria vaccine forms a crucial component of future malaria control and elimination tool.

Several research studies $[1,13,14,23,25,27,29,39-41,55]$ have focused on understanding the dynamics and control of P. falciparum infections. Although most of these models have investigated parameter importance in driving malaria infection, this is mainly based on the basic reproduction number of the model, which often do not have all the model parameters. Sensitivity analysis for the entire parameter space provides the right rigour for parameter evaluation in driving the disease progression. Previous studies [44,71], used parameter-driven LHS/PRCC procedure to ascertain important parameters and their relative importance in 
the disease model output. In [21], LHS/PRCC procedure is coupled with the optimal control numerical procedure to simultaneously examine the effects parameters on the objective functional value. In this paper, the LHS/PRCC uncertainty and sensitivity analysis applied to an in-host $P$. falciparum malaria model with vaccine controls. Moreover, multi-component and multi-stage vaccine combinations is evaluated to establish optimal vaccine combinations.

This paper is organised as follows: we provide details of the in-host malaria transmission model and its properties such as non-negativity and boundedness of solutions in "The Model" section. We carry out model analysis including model steady states and their stability conditions in "Model Analysis" section. In "Uncertainty and Sensitivity Analysis" section, we derive sensitivity indices of the basic reproduction number relative to model parameters. We also perform uncertainty and sensitivity analysis of model parameters subject to infective and infected state variables. The existence of model equilibrium points is numerically demonstrated in "Numerical Simulation" section. Numerical evaluation of multi-component and multi-stage vaccine cocktail completes "Numerical Simulation" section. A discussion on epidemiological implications of uncertainty, sensitivity analysis and vaccine combinations completes the paper in "Epidemiological Implications and Conclusion" section.

\section{The Model}

We consider an in-host $P$. falciparum malaria model. The model anayses the dynamics of interactions between the human red blood cells (erythrocytes), the liver hepatocytes, the malaria parasite (P. falciparum) and the cytotoxic $\mathrm{CD} 8^{+}$T-cells in the presence of multicomponent and multi-stage vaccine antigens. The compartmental deterministic model is an extension of in-most malaria model by the authors in [49]. The model is thus composed of nine compartments: the malaria sporozoites $S(t)$, the susceptible hepatocytes $H(t)$, the infected hepatocytes $X(t)$, the susceptible erythrocytes $R(t)$, the infected red blood cells $T(t)$, the malaria gametocytes $G(t)$, the malaria merozoites $M(t)$, unactivated CD ${ }^{+}$T-cells $W_{n}(t)$ and activated $\mathrm{CD} 8^{+} \mathrm{T}$-cells $W_{a}(t)$.

The activation of $\mathrm{CD} 8^{+}$T-cells is caused by the presence of infected hepatocytes and infected erythrocytes only. This activation is further assumed to occur at a constant rate $\lambda_{w}$ for both $X$ and $T$. Unactivated $C D 8^{+}$T-cells are produced at a fixed rate $\Omega$ from the thymus. Unlike the model in [49], we have assumed that a proportion $q$ of infected erythrocytes and a proportion $\rho$ of infected hepatocytes get eradicated by the CD ${ }^{+}$T-cells before they develop to the bursting stage. The rest of infected hepatocytes develop asexually and burst open to release blood stage merozoites. We assume that some of the newly generated merozoites develop into gametocytes. This proportion is denoted by the parameter $\pi$. The commitment to gametocytogenesis is presumably made during the immediately preceding cycle of blood schizogony [13]. These blood floating gametocytes are transmitted to the mosquito vector during feeding, marking the onset of the sporogonic cycle (not considered in this study).

During blood meal, infected mosquito injects $\Lambda$ sporozoites into the skin of the human host. The sporozoites traverse the skin and invade the hepatocytes $H$ at the rate $\beta_{s}$ to generate infected hepatocytes $X$ at the liver stage (see [64] for detailed description of the pre-erythrocytic stage infection). We assume a natural death rate of $\mu_{s}$ for malaria sporozoites. Although hepatocytes are generated at a rate $\lambda_{h}$ from the bone marrow, we assume that both the infected and uninfected hepatocytes experience natural death at the rates $\mu_{x}$ and $\mu_{h}$, respectively.

Three malaria vaccines are considered in this model to reduce the severity of $P$. falciparum malaria infection. These are: pre-erythrocytic vaccine (PEV) (see, $[6,7,46])$, blood stage 
Table 1 Description of the state variables

\begin{tabular}{ll}
\hline Variable & Description \\
\hline$W_{n}(t)$ & Concentration unactivated CD8 ${ }^{+}$T-cells \\
$W_{a}(t)$ & Concentration activated CD8 ${ }^{+}$T-cells \\
$H(t)$ & $\begin{array}{l}\text { Concentration of susceptible (uninfected) liver } \\
\text { hepatocytes }\end{array}$ \\
$X(t)$ & Concentration of infected hepatocytes \\
$S(t)$ & Population of malaria sporozoites \\
$R(t)$ & Concentration of susceptible (healthy) erythrocytes \\
$T(t)$ & Concentration of infected erythrocytes \\
$M(t)$ & Population of merozoites in blood \\
$G(t)$ & Concentration of gemetocytes in blood \\
\hline
\end{tabular}

vaccine (BSV) (see $[5,42,47]$ ), and transmission blocking vaccines (TBV) (see $[3,28]$ ). The leading PEV, BSV and TBV considered in this study are RTS,S/AS01, merozoite surface protein 3 (MSP3), P. falciparum ookinete surface antigens (Pfs25), respectively. The PEV, BSV and TBV vaccines are assumed to posses efficacies denoted by $\nu, \varrho$ and $\chi$, respectively, where $0<v<1,0<\varrho<1$ and $0<\chi<1$.

A summary of the descriptions of the state variables and model parameters is as shown in Tables 1 and 2 , respectively. Furthermore, the compartmental flow diagram for the inhost malaria model is provided in Fig. 1. We are motivated by the structural complexity of the in-host malaria model to apply LHS/PRCC technique for uncertainty and sensitivity analysis. The structural complexity in the model could lead to high levels of uncertainty in approximating the initial values of the input parameters.

Based on the dynamics described in Fig. 1, the following deterministic system describe the dynamics of the in-host malaria infections with multi-stage malaria vaccines:

$$
\begin{aligned}
\frac{\mathrm{d} S}{\mathrm{~d} t} & =(1-\chi) \Lambda-\mu_{s} S-\beta_{s} S H \\
\frac{\mathrm{d} H}{\mathrm{~d} t} & =\lambda_{h}-\mu_{h} H-\frac{\beta_{s}(1-v) S H}{1+d W_{a}}, \\
\frac{\mathrm{d} X}{\mathrm{~d} t} & =\frac{\beta_{s}(1-v) S H}{1+d W_{a}}-\mu_{x} X-\frac{\rho X W_{a}}{1+\varepsilon_{0} X}, \\
\frac{\mathrm{d} R}{\mathrm{~d} t} & =\lambda_{r}-\frac{(1-\varrho) \beta_{r} R M}{1+d W_{a}}-\mu_{r} R, \\
\frac{\mathrm{d} T}{\mathrm{~d} t} & =\frac{(1-\varrho) \beta_{r} R M}{1+d W_{a}}-\mu_{t} T-\frac{q T W_{a}}{1+\varepsilon_{1} T}, \\
\frac{\mathrm{d} M}{\mathrm{~d} t} & =\frac{P(1-q)(1-\pi)(1-a) \mu_{t} T}{1+d W_{a}}+(1-\rho)(1-b) N \mu_{x} X-\mu_{m} M-\beta_{r} R M, \\
\frac{\mathrm{d} G}{\mathrm{~d} t} & =\frac{P(1-q)(1-a) \pi \mu_{t} T}{1+d W_{a}}-\mu_{g} G, \\
\frac{\mathrm{d} W_{n}}{\mathrm{~d} t} & =\Omega-\tau \lambda_{w}(X+T) W_{n}-\mu_{n} W_{n}, \\
\frac{\mathrm{d} W_{a}}{\mathrm{~d} t} & =\tau \lambda_{w}(X+T) W_{n}-\mu_{a} W_{a},
\end{aligned}
$$


Table 2 Table showing model parameters and their descriptions

\begin{tabular}{|c|c|}
\hline Parameter symbol & Parameter description \\
\hline$P$ & $\begin{array}{l}\text { Number of merozoites released per bursting } \\
\text { erythrocyte }\end{array}$ \\
\hline$N$ & $\begin{array}{l}\text { Number of merozoites released per bursting } \\
\text { hepatocytes }\end{array}$ \\
\hline$\Lambda$ & Injection rate for sporozoites \\
\hline$\mu_{h}$ & Death rate of susceptible liver hepatocytes \\
\hline$\mu_{x}$ & Death rate of susceptible erythrocytes \\
\hline$\mu_{s} \mu_{m}$ & $\begin{array}{l}\text { Death rates of sporozoites and merozoites } \\
\text { respectively }\end{array}$ \\
\hline$\lambda_{h}$ & The recruitment rate of hepatocytes \\
\hline$\lambda_{r}$ & $\begin{array}{l}\text { The recruitment rate of erythrocytes from the } \\
\text { bone marrow }\end{array}$ \\
\hline$\pi$ & $\begin{array}{l}\text { A proportion of merozoites that develop into } \\
\text { gametocytes per dying infected red blood cell. }\end{array}$ \\
\hline$\rho$ & Immunosensitivity of infected hepatocytes \\
\hline$q$ & Immunosensitivity of infected erythrocytes \\
\hline$\beta_{S}, \beta_{r}$ & $\begin{array}{l}\text { Rate of infection of hepatocytes and } \\
\text { erythrocytes, respectively }\end{array}$ \\
\hline
\end{tabular}

$b$

A rate accounting for $\mathrm{PEV}$-induced reduction of merozoites released per bursting infected hepatocyte

\begin{tabular}{|c|c|}
\hline$\mu_{r}$ & Death rate of susceptible erythrocytes \\
\hline$\mu_{t}$ & Mortality rate of infected erythrocytes \\
\hline$\mu_{g}, \mu_{m}$ & $\begin{array}{l}\text { Mortality rate of gametocytes and merozoites, } \\
\text { respectively }\end{array}$ \\
\hline$\chi, \varrho, v$ & Vaccine efficacies \\
\hline$d$ & Inhibition rate of $\mathrm{CD}^{+} \mathrm{T}$-cells response \\
\hline$\Omega$ & $\begin{array}{l}\text { Recruitment rate of unactivated } \mathrm{CD}^{+}{ }^{+} \mathrm{T} \text {-cells } \\
\text { from the thymus }\end{array}$ \\
\hline$\lambda_{w}$ & $\begin{array}{l}\text { Immunogenicity of IRBCs and infected } \\
\text { hepatocytes }\end{array}$ \\
\hline $1 / \varepsilon_{0}$ & $\begin{array}{l}\text { A saturation (half) constant for infected } \\
\text { hepatocytes }\end{array}$ \\
\hline $1 / \varepsilon_{1}$ & $\begin{array}{l}\text { A saturation (half) constant for infected red } \\
\text { blood cells }\end{array}$ \\
\hline$\tau$ & $\begin{array}{l}\text { A rate accounting for vaccine-induced increased } \\
\text { generation of activated } \mathrm{CD} 8^{+} \mathrm{T} \text {-cells }\end{array}$ \\
\hline$\mu_{n}, \mu_{a}$ & $\begin{array}{l}\text { Death rates of unactivated and activated } \mathrm{CD}^{+} \\
\text {T-cells respectively }\end{array}$ \\
\hline$a$ & $\begin{array}{l}\text { A rate accounting for BSV-induced reduction of } \\
\text { merozoites released per bursting erythrocyte }\end{array}$ \\
\hline
\end{tabular}

with the general initial conditions:

$$
\begin{aligned}
& S(0) \geq 0, \quad H(0)>0, \quad X(0) \geq 0, \quad R(0)>0, \quad T(0) \geq 0, \quad M(0) \geq 0, \\
& G(0) \geq 0, \quad W_{n}(0)>0, \quad W_{a}(0) \geq 0 .
\end{aligned}
$$




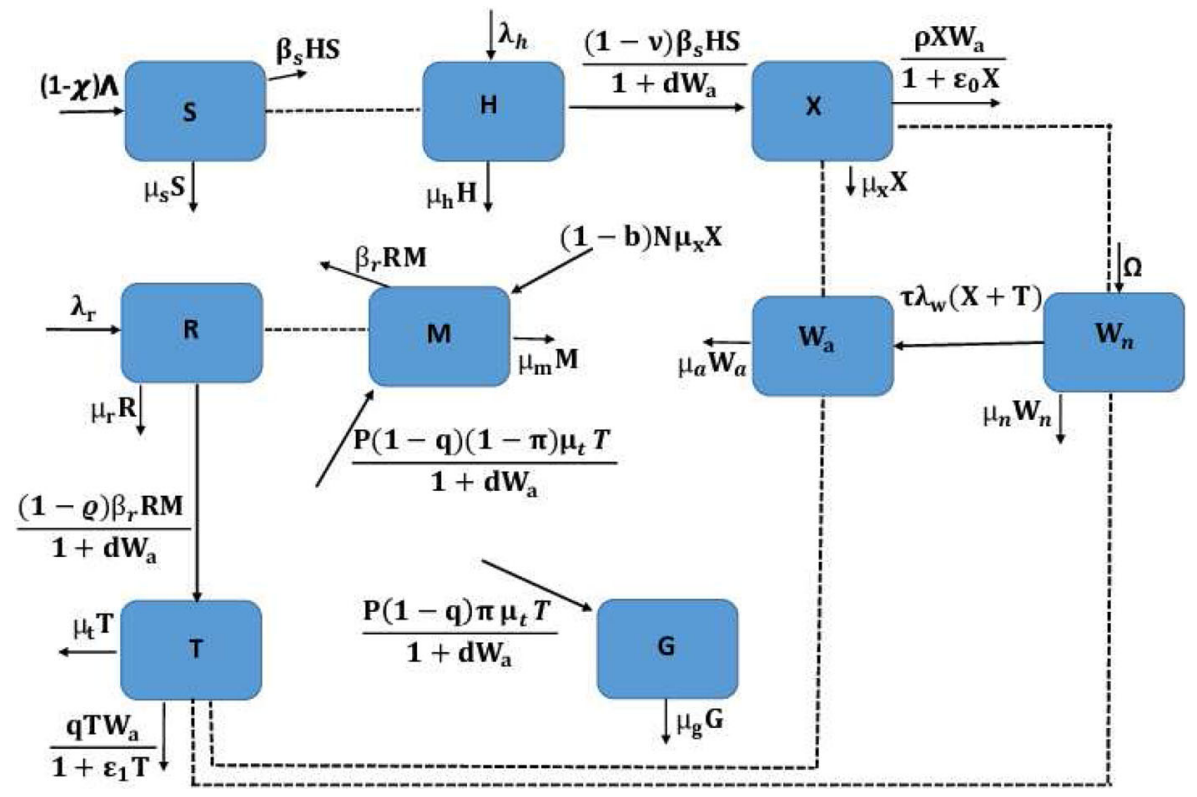

Fig. 1 A compartmental representation of the dynamics of in-host malaria infection with vaccine controls

\section{Properties of the Model}

\section{Positivity of Solutions}

The long term behaviour of any dynamical system is instrumental in the understanding of the system. The model system (1) monitors changes in the concentrations of blood cells, hepatocytes, malaria parasite and $\mathrm{CD}^{+} \mathrm{T}$-cells. To warrant biological sense, the state variables of system (1) should be non-negative. Specifically, we show that all solutions of model system (1) with non-negative initial conditions will remain non-negative for all time $t \geq 0$.

Theorem 1 Given non-negative initial conditions in Eq. (2), the in-host model (1) has a non-negative solution ( $\left.S(t), H(t), X(t), R(t), T(t), M(t), G(t), W_{n}(t), W_{a}(t)\right)$, for all time $t \geq 0$.

Proof From the first equation in system (1), we can clearly see that

$$
\frac{\mathrm{d} S}{\mathrm{~d} t} \geq-\left(\mu_{s}+\beta_{s} H\right) S .
$$

Upon integrating equation (3), we get

$$
S(t) \geq S_{0} \exp \left\{-\int_{0}^{t}\left(\mu_{s}+\beta_{s} H(s)\right) \mathrm{d} s\right\} .
$$

Therefore, $S(t) \geq 0$ for all $t \geq 0$.

Similarly, from the last equation in system (1), we have

$$
\frac{\mathrm{d} W_{a}}{\mathrm{~d} t} \geq-\mu_{a} W_{a} .
$$


Upon integrating the separable equation in (5), we obtain

$$
W_{a}(t)=W_{a}(0) \exp \left(-\mu_{a} t\right) \geq 0 .
$$

Using the same argument, the rest of the state variables can similarly be shown to be non-negative for all time $t \geq 0$. That is, $H(t)>0, X(t) \geq 0, R(t)>0, T(t) \geq 0, M(t) \geq 0$, $G(t) \geq 0$ and $W_{n}(t)>0 \forall t \geq 0$. This completes the proof.

\section{Boundedness of Solutions}

The non-negative solutions to model system (1) must be well posed in regions of biological sense. We therefore prove that the solutions are bounded.

Let $N_{r}$ be the sum of the density of susceptible and infected erythrocytes in the host. We thus have $N_{r}(t)=R(t)+T(t)$. Therefore,

$$
\begin{aligned}
\frac{\mathrm{d} N_{r}}{\mathrm{~d} t} & =\lambda_{r}-\mu_{r} R-\mu_{t} T-q T W_{a}, \\
& \leq \lambda_{r}-\hat{\mu} N_{r} \quad \text { where } \hat{\mu}=\min \left\{\mu_{r}, \mu_{t}\right\} .
\end{aligned}
$$

Using the initial condition $N_{r}(0)=N_{r 0}>0$, Eq. (7) is solved by integration factor method to obtain

$$
N_{r}(t) \leq \frac{\lambda_{r}}{\hat{\mu}}+e^{-\hat{\mu} t}\left(N_{r}(0)-\frac{\lambda_{r}}{\hat{\mu}}\right)
$$

This implies that at any time $t, N_{r}(t) \leq \max \left\{N_{r}(0), \frac{\lambda_{r}}{\hat{\mu}}\right\}$.

Again, let $N_{p}(t)=S(t)+M(t)+G(t)$, be the sum of malaria parasites at any time $t$ during clinical $P$. falciparum infection. So that,

$$
\begin{aligned}
\frac{\mathrm{d} N_{p}}{\mathrm{~d} t}= & (1-\chi) \Lambda+\frac{P(1-q)(1-\pi)(1-a) \mu_{t} T}{1+d W_{a}}+(1-\rho)(1-b) N \mu_{x} X \\
& +\frac{P(1-q)(1-a) \pi \mu_{t} T}{1+d W_{a}}-\mu_{s} S-\mu_{m} M-\mu_{g} G-\beta_{s} S H-\beta_{r} R M, \\
\leq & (1-\chi) \Lambda+\Psi_{1}(t)-\bar{\mu} N_{p} \quad \text { where } \bar{\mu}=\min \left\{\mu_{s}, \mu_{m}, \mu_{g}\right\}, \quad \text { and } \\
\Psi_{1}(t)= & \frac{P(1-q)(1-\pi)(1-a) \mu_{t} T}{1+d W_{a}}+(1-\rho)(1-b) N \mu_{x} X \\
& +\frac{P(1-q)(1-a) \pi \mu_{t} T}{1+d W_{a}} .
\end{aligned}
$$

Upon solving (8), we obtain:

$$
N_{p}(t) \leq \frac{(1-\chi) \Lambda}{\bar{\mu}}+e^{-\bar{\mu} t} \int_{0}^{t} e^{-\bar{\mu} t} \Psi_{1}(t) \mathrm{d} t+C_{1} e^{-\bar{\mu} t},
$$

where the constant of integration $C_{1}$ is given by,

$$
C_{1}=\left(N_{P}(0)-\frac{(1-\chi) \Lambda}{\bar{\mu}}\right)-\int_{0}^{s} \Psi_{1}(0) \mathrm{d} s .
$$

Observe that $N_{p}(t) \leq \max \left\{N_{p}(0), \frac{(1-\chi) \Lambda}{\bar{\mu}}\right\}$. The above procedure can similarly be applied to the total concentrations of the liver hepatocytes $N_{l}(t)=H(t)+X(t)$ and that of CD8 ${ }^{+}$T-cells $N_{w}(t)=W_{a}(t)+W_{n}(t)$ so that we arrive at: 


$$
\frac{\mathrm{d} N_{l}}{\mathrm{~d} t} \leq \lambda_{h}-\mu^{*} N_{l} \text { and } \frac{\mathrm{d} N_{w}}{\mathrm{~d} t} \leq \Omega-\mu_{w} N_{w}
$$

where $\mu^{*}=\min \left\{\mu_{h}, \mu_{x}\right\}$ and $\mu_{w}=\min \left\{\mu_{a}, \mu_{n}\right\}$.

Upon integrating and simplifying the equations in (10), we get,

$$
N_{l}(t) \leq \max \left\{N_{l}(0), \frac{\lambda_{h}}{\mu^{*}}\right\} \text { and } N_{w}(t) \leq \max \left\{N_{w}(0), \frac{\Omega}{\mu_{w}}\right\} \text { for all time } t \geq 0
$$

Therefore, all feasible solutions of in-host malaria model (1) enter the region:

$$
\begin{aligned}
\mathcal{D}= & \left\{(S, H, X, R, T, C, M, G, W) \in \mathbb{R}_{+}^{9}: N_{r}(t) \leq \max \left\{N_{r}(0), \frac{\lambda_{r}}{\hat{\mu}}\right\}\right. \\
& N_{p}(t) \leq \max \left\{N_{p}(0), \frac{(1-\chi) \Lambda}{\bar{\mu}}\right\}, \\
& \left.N_{l}(t) \leq \max \left\{N_{l}(0), \frac{\lambda_{h}}{\mu^{*}}\right\}, N_{w}(t) \leq \max \left\{N_{w}(0), \frac{\Omega}{\mu_{w}}\right\}\right\} .
\end{aligned}
$$

which is a positively invariant set of model system (1). This implies that all solutions in $\mathcal{D}$ remain in $\mathcal{D} \quad \forall t \geq 0$. It thus suffices to study the dynamics of model system (1) in $\mathcal{D}$.

\section{Model Analysis}

\section{Existence and Stability Analysis of Disease-Free Equilibrium Point (DFE)}

The disease-free equilibrium $E_{0}$ occurs when there is no $P$. falciparum infection within the human host. At $E_{0}, \Lambda=0$ and there are no activated $\mathrm{CD} 8^{+}-\mathrm{T}$ cells; that is, $W_{a}=0$. By setting the right hand-side of system (1) to zero, we obtain the following disease free equilibrium:

$$
E_{0}=\left(S^{0}, H^{0}, X^{0}, R^{0}, T^{0}, M^{0}, G^{0}, W_{n}^{0}, W_{a}^{0}\right)=\left(0, \frac{\lambda_{h}}{\mu_{h}}, 0, \frac{\lambda_{r}}{\mu_{r}}, 0,0,0, \frac{\Omega}{\mu_{n}}, 0\right)
$$

The disease-free steady state is very crucial in the understanding of in-host malaria infection dynamics. In order to eradicate in-host malaria, we have to identify and establish necessary conditions for $E_{0}$ to be stable. This stability could be achieved through the use of effective anti-malarial drugs such as ACT or by using highly efficacious Plasmodium falciparum malaria vaccines.

Next, we apply the next generation matrix (NGM) approach (see [14,63] for detailed explanation of this technique) to compute the basic reproduction number $R_{0}$ of the in-host model (1). The NGM method presumes the local stability of $E_{0}$ for $R_{0}<1$ and instability for $R_{0}>1 . R_{0}$ is the spectral radius of the next generation matrix $\left(\mathrm{FV}^{-1}\right)$ of system (1); that is, $R_{0}=\rho\left(\mathrm{FV}^{-1}\right)$. Adopting the matrix notations in [63], the matrices $\mathrm{F}$ and $\mathrm{V}$ are respectively given by: 


$$
\mathrm{F}=\left(\begin{array}{ccccccc}
0 & 0 & 0 & 0 & 0 & 0 & 0 \\
0 & \frac{(1-\nu) \beta_{s} \lambda_{h}}{\mu_{h}} & 0 & 0 & 0 & 0 & 0 \\
0 & 0 & 0 & \frac{(1-\varrho) \beta_{r} \lambda_{r}}{\mu_{r}} & 0 & 0 & 0 \\
0 & 0 & 0 & 0 & 0 & 0 & 0 \\
0 & 0 & 0 & 0 & 0 & 0 & 0 \\
0 & 0 & 0 & 0 & 0 & 0 & 0 \\
0 & 0 & 0 & 0 & 0 & 0 & 0
\end{array}\right)
$$

and

$$
\mathrm{V}=\left(\begin{array}{ccccccc}
\frac{\beta_{s} \lambda_{h}}{\mu_{h}}+\mu_{s} & 0 & 0 & 0 & 0 & 0 & 0 \\
0 & \mu_{x} & 0 & 0 & 0 & 0 & 0 \\
0 & 0 & \mu_{t} & 0 & 0 & 0 & 0 \\
0 & -(1-\rho)(1-b) N \mu_{x} & \mathcal{A}_{1} & \frac{\beta_{r} \lambda_{r}}{\mu_{r}}+\mu_{m} & 0 & 0 & 0 \\
0 & 0 & -P \pi(1-a)(1-q) \mu_{t} & 0 & \mu_{g} & 0 & 0 \\
0 & \frac{\tau \Omega \lambda_{w}}{\mu_{n}} & \frac{\tau \Omega \lambda_{w}}{\mu_{n}} & 0 & 0 & \mu_{n} & 0 \\
0 & -\frac{\tau \Omega \lambda_{w}}{\mu_{n}} & -\frac{\tau \Omega \lambda_{w}}{\mu_{n}} & 0 & 0 & 0 & \mu_{a}
\end{array}\right),
$$

where $\mathcal{A}_{1}=-P(1-\pi)(1-q)(1-a) \mu_{t}$.

Thus, the basic reproduction number $R_{0}$ is given by

$$
R_{0}=\rho\left(F V^{-1}\right)=\max \left\{R_{01}, R_{02}\right\}
$$

where

$$
\mathrm{R}_{01}=\frac{(1-v) \beta_{s} \lambda_{h}}{\mu_{h} \mu_{x}}
$$

and

$$
\mathrm{R}_{02}=\frac{P(1-\pi)(1-a)(1-\varrho)(1-q) \beta_{r} \lambda_{r}}{\beta_{r} \lambda_{r}+\mu_{m} \mu_{r}} .
$$

Observe that $R_{01}$ is the contribution of new cell invasions at the liver stage due to mortile sporozoites and $R_{02}$ is the contributions of new cell invasions at the blood stage due to merozoite parasites. From Eqs. (15) and (16), it is evident that new cell invasions occur both at the liver stage (as depicted by $R_{01}$ ) and at the blood stage (as depicted by $R_{02}$ ). This implies that clinical control of $P$. falciparum malaria should target the parasite at both stages of its life cycle within the human host. An anti-malarial drug with the capacity to eradicate the sporozoites at the pre-erythrocytic stage and merozoites at the erythrocytic stage is most recommended. Furthermore, malaria vaccine development should equally focus on both stages of parasite development. A novel malaria vaccine should begin parasite eradication from the pre-erythrocytic stage and this should continue to the blood stage. This result is very important to the ongoing research and development of malaria vaccines.

The global stability of the disease-free equilibrium is established using a suitable Lyapunov candidate function $[34,35,48]$. We thus have the following theorem.

Theorem 2 The disease-free equilibrium $E_{0}$ is globally asymptotically stable in $R_{+}^{9}$ if $R_{0}<$ 1.

Proof Let $V(X, T, S, M, G)=\psi_{1} X+\psi_{2} T+\psi_{3} S+\psi_{4} M+\psi_{5} G$ be a candidate Lyapunov function for some non-negative coefficients $\psi_{1}, \psi_{2}, \psi_{3}, \psi_{4}$ and $\psi_{5}$. The derivative of $V$ with respect to time is by 


$$
\begin{aligned}
\frac{\mathrm{d} V}{\mathrm{~d} t}= & \psi_{1} \frac{\mathrm{d} X}{\mathrm{~d} t}+\psi_{2} \frac{\mathrm{d} T}{\mathrm{~d} t}+\psi_{3} \frac{\mathrm{d} S}{\mathrm{~d} t}+\psi_{4} \frac{\mathrm{d} M}{\mathrm{~d} t}+\psi_{5} \frac{\mathrm{d} G}{\mathrm{~d} t} \\
= & \psi_{1}\left(\frac{\beta_{s}(1-v) S H}{1+d W_{a}}-\mu_{x} X-\frac{\rho X W_{a}}{1+\varepsilon_{0} X}\right)+\psi_{2}\left(\frac{(1-\varrho) \beta_{r} R M}{1+d W_{a}}-\mu_{t} T-\frac{q T W_{a}}{1+\varepsilon_{1} T}\right) \\
& +\psi_{3}\left((1-\chi) \Lambda-\mu_{s} S-\beta_{s} S H\right) \\
& +\psi_{4}\left(\frac{P(1-q)(1-\pi)(1-a) \mu_{t} T}{1+d W_{a}}+(1-\rho)(1-b) N \mu_{x} X-\mu_{m} M-\beta_{r} R M\right) \\
& +\psi_{5}\left(\frac{P(1-q)(1-a) \pi \mu_{t} T}{1+d W_{a}}-\mu_{g} G\right), \\
= & \left(-\mu_{x}\left(N \psi_{4}(b(-\rho)+b+\rho-1)+\psi_{1}\right)-\frac{\Omega}{\mu_{n}}\left(\rho \psi_{1}+q \psi_{2}\right)\right) X \\
& +\left(-\mu_{t}\left((a-1) P(q-1)\left((\pi-1) \psi_{4}-\pi \psi_{5}\right)+\psi_{2}\right)\right) T \\
& +\left(\psi_{3}\left(-\mu_{s}\right)-\frac{\lambda_{h}}{\mu_{h}}\left((v-1) \psi_{1}+\psi_{3}\right) \beta_{s}\right) S \\
& +\left(\psi_{4}\left(-\mu_{m}\right)-\frac{\lambda_{r}}{\mu_{r}}\left((e-1) \psi_{2}+\psi_{4}\right) \beta_{r}\right) M \\
& +\left(-\mu_{g} \psi_{5}\right) G .
\end{aligned}
$$

Setting the coefficients of $X, T, S, M$ and $G$ to zero and solving, we get

$$
\begin{aligned}
& \psi_{1}=\frac{N(1-b)(1-\rho) \mu_{x}(1-\varrho) \beta_{r} \lambda_{r} \mu_{a}^{2}-q \Omega\left(\beta_{r} \lambda_{r}+\mu_{m} \mu_{r}\right) \mu_{a}}{(1-\varrho) \beta_{r} \lambda_{r} \mu_{a}\left(\rho \Omega+\mu_{x} \mu_{a}\right)} \psi_{4}, \\
& \psi_{3}=\frac{\frac{\lambda_{h}}{\mu_{h}}(1-v) \beta_{s}}{\frac{\lambda_{h}}{\mu_{h}} \beta_{s}+\mu_{s}}\left[\frac{N(1-b)(1-\rho) \mu_{x}(1-\varrho) \beta_{r} \lambda_{r} \mu_{a}^{2}-q \Omega\left(\beta_{r} \lambda_{r}+\mu_{m} \mu_{r}\right) \mu_{a}}{(1-\varrho) \beta_{r} \lambda_{r} \mu_{a}\left(\rho \Omega+\mu_{x} \mu_{a}\right)}\right] \psi_{4}, \\
& \psi_{2}=\left[\frac{\frac{\lambda_{r}}{\mu_{r}} \beta_{r}+\mu_{m}}{(1-\varrho) \frac{\lambda_{r}}{\mu_{r}} \beta_{r}}\right] \psi_{4} \text { and } \psi_{5}=\frac{\frac{1}{1-\varrho}-P(1-a)(1-\pi)(1-q)+\frac{\mu_{m} \mu_{r}}{\beta_{r} \lambda_{r}(1-\varrho)}}{\pi P(1-a)(1-q)} \psi_{4} .
\end{aligned}
$$

Using these coefficients, the time derivatives of the Lyapunov function is given as

$$
\begin{aligned}
\frac{\mathrm{d} V}{\mathrm{~d} t}= & -\frac{\left.(q+(1-b)(1-\varrho) N(1-\rho) \rho) \beta_{r} \lambda_{r}+q \mu_{m} \mu_{r}\right)\left(\mu_{a}+\mu_{n}\right) \mu_{x}}{(1-\varrho) \beta_{r} \lambda_{r} \mu_{n}\left(\rho \Omega+\mu_{a} \mu_{x}\right)} X \\
& -\frac{\mu_{m} \mu_{r} \mu_{t}\left(1+(1-\varrho)^{2} \lambda_{r}^{2}\right)}{(1-e) \beta_{r} \lambda_{r}} T-\frac{\mu_{g}(1-\varrho) \lambda_{r} \mu_{m} \mu_{r}}{\pi \beta_{r}(1-a)(1-q)} G\left(1-R_{0}\right) .
\end{aligned}
$$

If $R_{0}<1$ in (17) then $\left[\mu_{g}(1-\varrho) \lambda_{r} \mu_{m} \mu_{r}\right] /\left[\pi \beta_{r}(1-a)(1-q)\right]\left(1-R_{0}\right)>0$. We thus obtain $\frac{\mathrm{d} V}{\mathrm{~d} t} \leq 0$. Furthermore, $\frac{\mathrm{d} V}{\mathrm{~d} t}=0$ if and only if $T=X=G=0$. Therefore, $V$ is a Lyapunov function in $\mathcal{D}$. Given that $\mathcal{D}$ is positively invariant and attracting, it follows that the maximum invariant set in $\left\{\left(S, H, X, R, T, M, G, W_{n}, W_{a}\right) \in \mathcal{D}: \dot{V}=0\right\}$ is the singleton $E_{0}$. By LaSalle's Invariant principle [33], $E_{0}$ is globally asymptotically stable in $\mathcal{D}$ provided that $R_{0}<1$.

\section{The Malaria Persistent Steady State}

We establish the malaria persistent equilibrium $E_{p}$ for model system (1) in the presence of $\mathrm{CD}^{+}$T-cells by equating the right hand side of system (1) to zero. Using Mathematica software [31], model system (1), exhibits a malaria-persistent equilibrium point 


$$
E_{p}=\left(S^{\dagger}, H^{*}, X^{*}, R^{*}, T^{*}, M^{*}, G^{*}, W_{n}^{*}, W_{a}^{*}\right)
$$

where

$$
\begin{aligned}
& H^{*}=\frac{\left(1+d W_{a}^{*}\right) \lambda_{h}}{(1-v) \beta_{s} S^{*}+\left(1+d W_{a}^{*}\right) \mu_{h}}, \\
& R^{*}=\frac{\left(1+d W_{a}^{*}\right) \lambda_{r}}{(1-\varrho) \beta_{r} M^{*}+\left(1+d W_{a}^{*}\right) \mu_{r}}, \\
& W_{n}^{*}=\frac{\Omega}{\tau \lambda_{w}\left(X^{*}+T^{*}\right)+\mu_{n}}, \\
& W_{a}^{*}=\frac{\tau \lambda_{w}\left(X^{*}+T^{*}\right) W_{n}^{*}}{\mu_{a}}, \\
& G^{*}=\frac{P(1-q)(1-a) \pi \mu_{t} T^{*}}{\mu_{g}\left(1+d W_{a}^{*}\right)}, \\
& M^{*}=\frac{\left(1+d W_{a}^{*}\right)\left[(1-b)^{2}(1-\varrho) N X^{*} \beta_{r} \mu_{m} \mu_{x}-\left(1+d W_{a}^{*}\right) \mu_{r}\right] \pm \sqrt{\Delta}}{2(1-\varrho)\left(1+d W_{a}^{*}\right) \beta_{r}}, \text { where } \\
& \Delta_{m}=\left(1+d W_{a}^{*}\right)\left\{\left(-(e-1) \beta_{r}\left(-4(1-\pi)(1-a) P(1-q) T^{*} \mu_{t}\right.\right.\right. \\
& \left.+(1-b)^{2} N X^{*}\left(1+d W_{a}^{*}\right) \beta_{r} \mu_{x}\left(4\left(1+d W_{a}^{*}\right) \lambda_{r}+(1-b)^{2}(1-e) N X^{*} \mu_{m}^{2} \mu_{x}\right)\right) \\
& \left.-2(1-b)^{2}(1-e) N X^{*}\left(1+d W_{a}^{*}\right)^{2} \mu_{m} \beta_{r} \mu_{r} \mu_{x}+\left(1+d W_{a}^{*}\right)^{3} \mu_{r}^{2}\right\} \text {, } \\
& S^{*}=\frac{1}{-2(1-v) \beta_{s} v_{s}}\left\{-\Delta_{s} \pm \sqrt{\Delta_{s}^{2}+4\left((1-v) \beta_{s} \mu_{s}\right)\left((1-\chi) \Lambda\left(1+d W_{a}^{*}\right) \mu_{h}\right)}\right\} \text {, } \\
& \text { where } \Delta_{s}=\beta_{s}\left(\Lambda(1-\chi)(1-v)-\left(1+d W_{a}^{*}\right) \lambda_{h}\right)-\left(1+d W_{a}^{*}\right) \mu_{h} \mu_{s}, \\
& T^{*}=\frac{1}{-2 \epsilon_{1}\left((1-\varrho) \beta_{r} M^{*}+\left(1+d W_{a}^{*}\right) \mu_{r}\right) \mu_{t}}\left\{-\Delta_{t} \pm \sqrt{\Delta_{T}^{*}}\right\}, \\
& \text { where } \Delta_{t}=\beta_{s}\left(\Lambda(1-\chi)(1-v)-\left(1+d W_{a}^{*}\right) \lambda_{h}\right)-\left(1+d W_{a}^{*}\right) \mu_{h} \mu_{s} \text {; } \\
& \Delta_{T}^{*}=\Delta_{t}^{2}+4 \epsilon_{1} \mu_{t}\left((1-\varrho) \beta_{r} M^{*}+\left(1+d W_{a}^{*}\right) \mu_{r}\right)\left(\beta_{r} \lambda_{r}(1-\varrho) M^{*}\right) \text { and } \\
& X^{*}=\frac{\Delta_{x} \pm \sqrt{\left.\Delta_{x}^{2}+4 \epsilon_{0}\left((1-v) \beta_{s} S^{*}+\left(1+d W_{a}^{*}\right) \mu_{h}\right) \mu_{x}\right)(1-v) \beta_{s} \lambda_{h} S^{*}}}{2 \epsilon_{0}\left((1-v) \beta_{S} S^{*}+\left(1+d W_{a}^{*}\right) \mu_{h}\right) \mu_{x}} \text {, where } \\
& \Delta_{x}=(1-v)\left(\epsilon_{0} \lambda_{h}-\mu_{x}\right) \beta_{s} S^{*}-d \rho W_{a}^{* 2} \mu_{h}-\mu_{h} \mu_{x} \\
& -W_{a}^{*}\left((1-v) \rho \beta_{s} S^{*}+\mu_{h}\left(\rho+d \mu_{x}\right)\right) \text {. }
\end{aligned}
$$

Based on the formulated model in Eq. (1), the activated CD8 ${ }^{+}$T-cells only exists due to the presence of infected erythrocytes and infected liver hepatocytes. Hence, in the absence of malaria infection $(X=T=0), W_{a}=0$. Moreover, non of the malaria parasites exists; that is $S^{*}=M^{*}=G^{*}=0$. We further observe that when $S^{*}=M^{*}=X^{*}=T^{*}=W_{a}^{*}=0$, the susceptible erythrocytes, susceptible hepatocytes and the unactivated CD ${ }^{+}$T-cells become $H^{*}=\frac{\lambda_{h}}{\mu_{h}}, R^{*}=\frac{\lambda_{r}}{\mu_{r}}$ and $W_{n}^{*}=\frac{\Omega}{\mu_{n}}$ respectively. We thus arrive at an equilibrium point called the disease free equilibrium point of model (1) illustrated in Eq. (12). From the above computations, it is evident that the analytical expressions of $E_{p}$ is too complex. We shall therefore provide a numerical illustration of the model equilibrium points in "Numerical Simulation" section.

Theorem 3 The malaria persistent steady state $E_{p}$ is locally asymptotically stable if $R_{0}>1$ in the interior of the biologically feasible region $\mathcal{D}$. 
Proof The stability of the malaria persistent equilibrium $E_{p}$ (when $R_{0}>1$ but close to 1 ) is proved using the center manifold theory (CMT) presented by Castillo-Chavez and Song in [11]. Adopting the notations used in [11] and without re-stating the theorem, we calculate the values of arguments $\mathbf{a}$ and $\mathbf{b}$. The signs (+ or - ) of $\mathbf{a}$ and $\mathbf{b}$ determine the local dynamics of system (1) around $E_{p}$ [66].

Suppose we consider $\beta_{*}$ to be our bifurcation parameter, then at $R_{0}=1$, we have $\beta_{*}=$ $\max \left\{\beta_{s}^{*}, \beta_{r}^{*}\right\}$, where

$$
\beta_{r}^{*}=\frac{\mu_{m} \mu_{r}}{[P(1-q)(1-\pi)(1-\varrho)(1-a)-1] \lambda_{r}} \quad \text { and } \quad \beta_{s}^{*}=\frac{\mu_{h} \mu_{x}}{(1-v) \lambda_{h}} .
$$

In applying the CMT, we begin by transforming the state variables $(S, H, X, R, T, M, G$, $\left.W_{n}, W_{a}\right)$ into $\left(x_{1}, x_{2}, x_{3}, x_{4}, x_{5}, x_{6}, x_{7}, x_{8}, x_{9}\right)$ respectively. The model system (1), is hence represented as $\frac{d g}{d t}=g(\mathbf{x})$, where $\mathbf{x}=\left(x_{1}, x_{2}, x_{3}, x_{4}, x_{5}, x_{6}, x_{7}, x_{8}, x_{9}\right)$. The model system (1) thus becomes

$$
\begin{aligned}
& \dot{x}_{1}=(1-\chi) \Lambda-\mu_{s} x_{1}-\beta_{s} x_{1} x_{2}, \\
& \dot{x}_{2}=\lambda_{h}-\mu_{h} x_{2}-\frac{\beta_{s}(1-v) x_{1} x_{2}}{1+d x_{9}}, \\
& \dot{x}_{3}=\frac{\beta_{s}(1-v) x_{1} x_{2}}{1+d x_{9}}-\mu_{x} x_{3}-\frac{\rho x_{3} x_{9}}{1+\varepsilon_{0} x_{3}}, \\
& \dot{x}_{4}=\lambda_{r}-\frac{(1-\varrho) \beta_{r} x_{4} x_{6}}{1+d x_{9}}-\mu_{r} x_{4}, \\
& \dot{x}_{5}=\frac{(1-\varrho) \beta_{r} x_{4} x_{6}}{1+d x_{9}}-\mu_{t} x_{5}-\frac{q x_{5} x_{9}}{1+\varepsilon_{1} x_{5}}, \\
& \dot{x}_{6}=\frac{P(1-q)(1-\pi)(1-a) \mu_{t} x_{5}}{1+d x_{9}}+(1-\rho)(1-b) N \mu_{x} x_{3}-\mu_{m} x_{6}-\beta_{r} x_{4} x_{6}, \\
& \dot{x}_{7}=\frac{P(1-q)(1-a) \pi \mu_{t} x_{5}}{1+d x_{9}}-\mu_{g} x_{7}, \\
& \dot{x}_{8}=\Omega-\tau \lambda_{w}\left(x_{3}+x_{5}\right) x_{8}-\mu_{n} x_{8}, \\
& \dot{x}_{9}=\tau \lambda_{w}\left(x_{3}+x_{5}\right) x_{8}-\mu_{a} x_{9},
\end{aligned}
$$

such that;

$x_{1}(0) \geq 0, x_{2}(0) \geq 0, x_{3}(0) \geq 0, x_{4}(0) \geq 0, x_{5}(0) \geq 0, x_{6}(0) \geq 0, x_{7}(0) \geq 0, x_{8}(0) \geq 0$, $x_{9}(0) \geq 0$.

The new model system (28) with the bifurcation point $\beta_{*}$ has a simple zero eigenvalue. This attribute enables us to use the CMT to analyze the stability of system (28) near $\beta_{*}=$ $\max \left\{\beta_{s}^{*}, \beta_{r}^{*}\right\}=\max \left\{\beta_{s}, \beta_{r}\right\}$.

Therefore, a right eigenvector $w=\left[w_{1}, w_{2}, w_{3}, w_{4}, w_{5}, w_{6}, w_{7}, w_{8}, w_{9}\right]^{T}$ associated with the simple zero eigenvalue is obtained from the linearization matrix corresponding to the zero eigenvector as follows: 


$$
\left(\begin{array}{ccccccccc}
-A_{2} & 0 & 0 & 0 & 0 & 0 & 0 & 0 & 0 \\
-A_{3} & -\mu_{h} & 0 & 0 & 0 & 0 & 0 & 0 & \\
A_{3} & 0 & -\mu_{x} & 0 & 0 & 0 & 0 & 0 & 0 \\
0 & 0 & 0 & -\mu_{r} & 0 & -A_{4} & 0 & 0 & 0 \\
0 & 0 & 0 & 0 & -\mu_{t} & A_{4} & 0 & 0 & 0 \\
0 & 0 & 0 & 0 & A_{0} & -A_{5} & 0 & 0 & 0 \\
0 & 0 & 0 & 0 & A_{1} & 0 & -\mu_{g} & 0 & 0 \\
0 & 0 & -\frac{\tau \Omega \lambda_{w}}{\mu_{n}} & 0 & -\frac{\tau \Omega \lambda_{w}}{\mu_{n}} & 0 & 0 & -\mu_{n} & 0 \\
0 & 0 & \frac{\tau \Omega \lambda_{w}}{\mu_{n}} & 0 & \frac{\tau \Omega \lambda_{w}}{\mu_{n}} & 0 & 0 & 0 & -\mu_{a}
\end{array}\right)\left(\begin{array}{l}
w_{1} \\
w_{2} \\
w_{3} \\
w_{4} \\
w_{5} \\
w_{6} \\
w_{7} \\
w_{8} \\
w_{9}
\end{array}\right)=\left(\begin{array}{l}
0 \\
0 \\
0 \\
0 \\
0 \\
0 \\
0 \\
0 \\
0
\end{array}\right)
$$

where $A_{0}=(1-a) P(1-\pi)(1-q) \mu_{t}, A_{1}=(1-a) P \pi(1-q) \mu_{t}, A_{2}=\left(\frac{\beta_{s} \lambda_{h}}{\mu_{h}}+\mu_{s}\right)$, $A_{3}=\frac{(1-v) \beta_{s} \lambda_{h}}{\mu_{h}}, A_{4}=\frac{(1-\varrho) \beta_{r} \lambda_{r}}{\mu_{r}}$ and $A_{5}=\left(\frac{\beta_{r} \lambda_{r}}{\mu_{r}}+\mu_{m}\right)$.

Solving for the right eigenvectors, we obtain

$$
\begin{aligned}
w_{5}= & \frac{-\left(\frac{\beta_{r} \lambda_{r}}{\mu_{r}}+\mu_{m}\right)}{(1-a) P(1-\pi)(1-q) \mu_{t}}, \quad w_{7}=\frac{-(1-a) P \pi(1-q) \mu_{t}}{\mu_{g}} \\
& +\frac{\left(\frac{\beta_{r} \lambda_{r}}{\mu_{r}}+\mu_{m}\right)}{(1-a) P(1-\pi)(1-q) \mu_{t}}, \\
w_{4}= & -\frac{(1-\varrho) \beta_{r} \lambda_{r}}{\mu_{r}^{2}}, \quad w_{8}=\frac{\tau \Omega \lambda_{w}}{(1-a) P(1-\pi)(1-q) \mu_{t} \mu_{n}^{2}}\left(\frac{\beta_{r} \lambda_{r}}{\mu_{r}}+\mu_{m}\right), w_{6}=1, \\
w_{9}= & -\frac{\tau \Omega \lambda_{w}}{\mu_{a} \mu_{n}}\left(\frac{\beta_{r} \lambda_{r}}{\mu_{r}}+\mu_{m}\right), \quad w_{1}=w_{2}=w_{3}=0 .
\end{aligned}
$$

Similarly, upon solving for the left eigenvector $v=\left[v_{1}, v_{2}, v_{3}, v_{4}, v_{5}, v_{6}, v_{7}, v_{8}, v_{9}\right]^{T}$ associated with the zero eigenvalue in system (28), we obtain

$$
v_{1}=v_{2}=v_{3}=v_{4}=0, \quad v_{5}=P(1-a)(1-\pi)(1-q), v_{6}=1, v_{7}, v_{8}, v_{9}=0 .
$$

Next, we calculate the values of $\mathbf{a}$ and $\mathbf{b}$ as described in [11]. That is,

$$
\mathbf{a}=\sum_{i, j, k=1}^{9} v_{k} w_{i} w_{j} \frac{\partial^{2} g_{k}(\mathbf{O}, 0)}{\partial x_{i} \partial x_{j}} \text { and } \mathbf{b}=\sum_{i, k=1}^{9} v_{k} w_{i} \frac{\partial^{2} g_{k}(\mathbf{O}, 0)}{\partial x_{i} \partial \beta_{*}}
$$

The non-zero partial derivatives of $g(x)$ in system (28) associated with the argument a are given as

$$
\begin{aligned}
\frac{\partial^{2} g_{1}}{\partial x_{1} \partial x_{2}} & =-\beta_{s}, \quad \frac{\partial^{2} g_{2}}{\partial x_{1} \partial x_{2}}=-(1-v) \beta_{s}, \quad \frac{\partial^{2} g_{2}}{\partial x_{1} \partial x_{9}}=d(1-v) \frac{\lambda_{h}}{\mu_{h}} \beta_{s}, \\
\frac{\partial^{2} g_{4}}{\partial x_{4} \partial x_{6}} & =-(1-\varrho) \beta_{r}, \\
\frac{\partial^{2} g_{4}}{\partial x_{6} \partial x_{9}} & =\frac{d(1-\varrho) \beta_{r} \lambda_{r}}{\mu_{r}}, \quad \frac{\partial^{2} g_{5}}{\partial x_{5} \partial x_{9}}=-q, \quad \frac{\partial^{2} g_{5}}{\partial x_{4} \partial x_{6}}=(1-\varrho) \beta_{r}, \\
\frac{\partial^{2} g_{5}}{\partial x_{6} \partial x_{9}} & =\frac{-d(1-\varrho) \beta_{r} \lambda_{r}}{\mu_{r}}, \\
\frac{\partial^{2} g_{6}}{\partial x_{4} \partial x_{6}} & =-\beta_{r}, \quad \frac{\partial^{2} g_{6}}{\partial x_{5} \partial x_{9}}=P(1-a)(1-\pi)(1-q) d \mu_{t},
\end{aligned}
$$




$$
\begin{aligned}
\frac{\partial^{2} g_{7}}{\partial x_{5} \partial x_{9}} & =P \pi(1-a)(1-q), \quad \frac{\partial^{2} g_{8}}{\partial x_{3} \partial x_{8}}=\frac{\partial^{2} g_{8}}{\partial x_{5} \partial x_{8}}=-\tau \lambda_{w}, \\
\frac{\partial^{2} g_{9}}{\partial x_{3} \partial x_{8}} & =\frac{\partial^{2} g_{9}}{\partial x_{5} \partial x_{8}}=\tau \lambda_{w} .
\end{aligned}
$$

Thus, the expression for $\mathbf{a}$ is:

$$
\begin{aligned}
\mathbf{a}= & v_{5} w_{5} w_{9} \frac{\partial^{2} g_{5}}{\partial x_{5} \partial x_{9}}+v_{5} w_{4} w_{6} \frac{\partial^{2} g_{5}}{\partial x_{4} \partial x_{6}}+v_{5} w_{6} w_{9} \frac{\partial^{2} g_{5}}{\partial x_{6} \partial x_{9}}+v_{6} w_{4} w_{6} \frac{\partial^{2} g_{6}}{\partial x_{4} \partial x_{6}} \\
& +v_{6} w_{5} w_{9} \frac{\partial^{2} g_{6}}{\partial x_{5} \partial x_{9}} \\
= & -\left(\frac{\beta_{r} \lambda_{r}}{\mu_{r}}+\mu_{m}\right)\left(\frac{d(1-\varrho) \beta_{r} \lambda_{r}}{\mu_{r}}\right)\left(\frac{P(1-a)(1-\pi)(1-q) \mu_{t} \tau \Omega \lambda_{w}}{\mu_{a} \mu_{t} \mu_{n}}\right)<0 .
\end{aligned}
$$

We repeat the above procedure in computing the values of $\mathbf{b}$. The non-zero partial derivatives of $g(x)$ associated with $\mathbf{b}$ are given by

$$
\begin{aligned}
\frac{\partial^{2} g_{1}}{\partial x_{1} \partial \beta_{*}} & =-\frac{\lambda_{h}}{\mu_{h}}, \quad \frac{\partial^{2} g_{2}}{\partial x_{1} \partial \beta_{*}}=\frac{-(1-v) \lambda_{h}}{\mu_{h}}, \quad \frac{\partial^{2} g_{3}}{\partial x_{1} \partial \beta_{*}}=\frac{(1-v) \lambda_{h}}{\mu_{h}}, \\
\frac{\partial^{2} g_{4}}{\partial x_{6} \partial \beta_{*}} & =\frac{-(1-\varrho) \lambda_{r}}{\mu_{r}}, \\
\frac{\partial^{2} g_{5}}{\partial x_{6} \partial \beta_{*}} & =\frac{(1-\varrho) \lambda_{r}}{\mu_{r}}, \quad \frac{\partial^{2} g_{6}}{\partial x_{6} \partial \beta_{*}}=\frac{-\lambda_{r}}{\mu_{r}} .
\end{aligned}
$$

Upon substituting these derivatives into Eq. (30), it follows that

$$
\begin{aligned}
\mathbf{b} & =v_{5} w_{6} \frac{\partial^{2} g_{5}}{\partial x_{6} \partial \beta_{*}}+v_{6} w_{6} \frac{\partial^{2} g_{6}}{\partial x_{6} \partial \beta_{*}} \\
& =\frac{\lambda_{r}}{\mu_{r}}(P(1-a)(1-\pi)(1-q)(1-\varrho)+1)>0 .
\end{aligned}
$$

Thus, $a$ is positive $(a>0)$ and $b$ is negative $(b<0)$. Based on item (iv) of theorem 2 in [11], the malaria persistent equilibrium $E_{p}$ is locally asymptotically stable for $R_{0}>1$, but close to 1 .

\section{Uncertainty and Sensitivity Analysis}

\section{Sensitivity Analysis of Infected and Infective States of the In-Host Malaria Model}

The in-host malaria model in this paper has numerous unknown parameters, coupled with limited data. Therefore, there is a considerable uncertainty [9] in estimating the values of the 30 transmission parameters in model system (1). The uncertainty in the input values allows significant variability in the model predictions of the future malaria parasite density within the human host and the concentrations of the infected red blood cells and hence the severity of clinical $P$. falciparum malaria. Consequently, we perform the LHS/PRCC sensitivity analysis to evaluate variabilities in model predictions. With LHS/PRCC technique, we are able to explore the entire parameter space of model (1), with minimal computer simulation.

We simultaneously examine the effects of the LHS parameters on the two infected state variables: the infected liver hepatocytes $X(t)$ and the infected erythrocytes $T(t)$ and the 
three infective parasite states: sporozoite $S(t)$, merozoites $M(t)$ and Gametocytes $G(t)$ in model system (1). The PRCCs are used to identify the key parameters contributing to the imprecision in predicting the future density of infected liver hepatocytes and infected red blood cells [9].

The process of performing LHS on disease models is described elsewhere $[8,38]$ and references cited therein. Following PRCC methodology described in [52], we rank the LHS parameters in the LHS matrix together with the outcome measures: $T(t), X(t), M(t), G(t)$ and $S(t)$. Two linear regression models are generated in response to each parameter and outcome measure. A Pearson rank correlation coefficient for the residuals from the two regression models gives the PRCC values for that specific parameter. Due to lack of data on the distribution function, we consider a uniform distribution for all model parameters in Table 3.

\section{Results of Analyzing the LHS/PRCC for the In-Host Malaria Model}

Using 1000 runs of Latin hypercube sampling, the PRCC and $p$ value data from the 30 parameters in model system (1) are summarized in Appendices A, B, C, D and E. The PRCC and $p$ values are computed based on the regression coefficient for outcome measures $(T(t), X(t), M(t), S(t)$ and $G(t)$ and displayed in Tables 5, 6, 78 and 9, respectively. The size of PRCC shows the importance of the uncertainty in estimating the value of the specific variable in contributing to the prediction imprecision [9]. The PRCC sign (+ or - ), however, shows the qualitative relationship between the input parameter and the output variables. Parameters with large PRCC values (PRCC from 0.5 to 1 or PRCC from -1 to $-0.5)$ coupled with a corresponding small $p$ value $(p$ value $<0.05)$ are considered as the most influential [21].

A slight change in a highly influential parameter is likely to produce a significant change in the outcome variable. The symbol (*) indicates less influential parameters with $p$ values $<0.05$ and PRCC values in the range $(-0.1$ to -0.49 or 0.1 to 0.49$)$. Further, the symbol $(* *)$ is used to indicate moderately critical parameters in prediction imprecision, and have $p$ value $<0.05$ and PRCC values in the range $(-0.5$ to -0.79 or 0.5 to 0.79$)$. Lastly, the symbol $(* * *)$ indicates the most critical and likely contributors to uncertainty, with PRCC values in the range $(-0.8$ to -0.99 or +0.8 to +0.99$)$.

Results in Appendices A, B, C, D and E indicate that the rate of invasion by merozoites $\beta_{r}$, the rate of invasion of liver hepatocytes $\beta_{s}$, malaria vaccine efficacies $(\varrho, v$, and $\chi)$ and the recruitment rates for the hepatocyte and erythrocytes $\lambda_{h}$ and $\lambda_{r}$ respectively are statistically significant in determining the variation of concentrations of erythrocytes, infected hepatocytes and the density of malaria parasites (sporozoites, merozoites and gametocytes). The infected erythocyte burst size $P$ and the number of merozoites released per bursting sporozoite $N$ is shown to significantly increase the the density of the merozoites and the concentration of infected red blood cells $T$ in blood. Clinical malaria controls should thus target these infected cells for eradication before they mature to bursting stage.

Moreover, from Appendices A and D, the proportion of merozoites that develop into gametocytes per dying infected erythrocyte $\pi$ and immunosensitivity of infected erythrocytes $q$ are shown to have significant influence in determining the concentrations of gametocytes and merozoites, respectively, during clinical P. falciparum infection. An efficacious blood stage vaccine coupled with a transmission blocking vaccine antigens has the potential of reducing the density of gametocytes during clinical malaria. This reduces parasite transmission to mosquito vector. 
Table 3 Table showing the numerical values of model parameter values used in sensitivity analysis and numerical simulations

\begin{tabular}{|c|c|c|c|c|c|}
\hline Symbol & Value & Min & Max & Unit & Source \\
\hline$\Lambda$ & 30 & 20 & 35 & Sporozoites/day & {$[56]$} \\
\hline$\mu_{s}$ & 1.2 & 0.8 & 1.5 & /day & [56] \\
\hline$\lambda_{r}$ & $3 \mathrm{E} 5$ & $3 \mathrm{E} 3$ & 3E6 & cells/ml/day & [36] \\
\hline$\lambda_{h}$ & $3 \mathrm{E} 5$ & $3 \mathrm{E} 3$ & $3 \mathrm{E} 6$ & cells $/ \mu 1^{-1} /$ day & [61] \\
\hline$\beta_{r}$ & $8 \mathrm{E}-5$ & $8 \mathrm{E}-7$ & $8 \mathrm{E}-3$ & $/ \mathrm{mm}^{3} /$ day & {$[15]$} \\
\hline$\beta_{S}$ & $5 \mathrm{E}-4$ & $5 \mathrm{E}-5$ & $5 \mathrm{E}-3$ & $/ \mathrm{mm}^{3} /$ day & {$[56]$} \\
\hline$\mu_{h}$ & 0.029 & 0.01 & 0.03 & /day & [49] \\
\hline$\mu_{x}$ & 0.02 & 0.01 & 0.05 & /day & {$[56]$} \\
\hline$\pi$ & 0.2 & 0.1 & 0.9 & Unitless & [59] \\
\hline$\mu_{r}$ & $8.3 \mathrm{E}-5$ & $8.3 \mathrm{E}-6$ & 0.0001 & $/$ day & {$[1]$} \\
\hline$\mu_{m}$ & 48 & 46 & 49 & /day & {$[36]$} \\
\hline$\mu_{t}$ & 0.7 & 0.5 & 0.8 & /day & [37] \\
\hline$\mu_{g}$ & 0.0625 & 0.5 & 0.78 & /day & {$[56]$} \\
\hline$\chi$ & 0.6 & 0 & 1 & Unitless & Assumed \\
\hline$\varrho$ & 0.45 & 0 & 1 & Unitless & {$[6]$} \\
\hline$v$ & 0.7 & 0 & 1 & Unitless & Assumed \\
\hline$d$ & $5 \mathrm{E}-4$ & $1 \mathrm{E}-4$ & 0.001 & Unitless & Assumed \\
\hline$\tau$ & 1.5 & 1.2 & 1.9 & Unitless & {$[45]$} \\
\hline$\lambda_{w}$ & 30 & 25 & 35 & /unitless/day & {$[45]$} \\
\hline$b$ & 0.17 & 0.2 & 0.5 & Unitless & {$[45]$} \\
\hline$a$ & 0.16 & 0.2 & 0.4 & Unitless & {$[45]$} \\
\hline$P$ & 16 & 13 & 20 & /erythrocytes/day & {$[13]$} \\
\hline$N$ & 10,000 & 8000 & 11,000 & /day & {$[60]$} \\
\hline$\epsilon_{0}$ & $1 E-5$ & $1 E-6$ & $1 \mathrm{E}-4$ & /day & {$[60]$} \\
\hline$\epsilon_{1}$ & $1 E-5$ & $1 \mathrm{E}-6$ & $1 \mathrm{E}-4$ & /day & {$[60]$} \\
\hline$q$ & 0.01 & 0.001 & 0.02 & Unitless & {$[45]$} \\
\hline$\rho$ & 0.3 & 0.2 & 0.45 & Unitless & {$[45]$} \\
\hline$\mu_{n}$ & 0.4 & 0.3 & 0.72 & /day & {$[1]$} \\
\hline$\mu_{a}$ & 0.4 & 0.3 & 0.72 & /day & {$[36]$} \\
\hline$\Omega$ & 30 & 24 & 33 & Unitless & [37] \\
\hline
\end{tabular}

Figures 2 and 3 illustrates Monte Carlo simulations for some of the parameters with the greatest PRCC values against model $R_{0}$.

It is evident that a highly efficacious pre-erythrocytic vaccine such as RTS,S/SA01 (with efficacy $v$ ) and blood stage vaccine such as MSP3 (with efficacy $\varepsilon$ ) could effectively help in reducing parasitemia and malaria severity during clinical $P$. falciparum infections. Sporozoite invasion rate $\beta_{s}$ and the merozoite invasion parameter $\beta_{r}$ are also shown to increase the model $R_{0}$ and hence the severity of the disease within the human host.

Although the LHS/PRCC technique is useful in identifying significant parameters in the in-host malaria model, it fails to rank the parameters in order of their contribution to variations and disease progression. This ranking is very crucial in the design of clinical interventions 


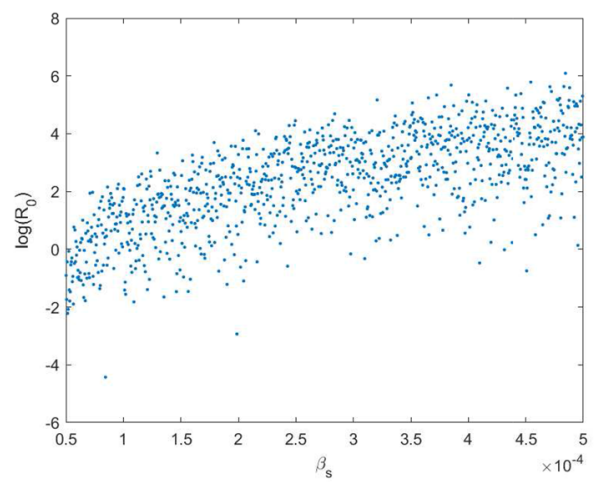

(a)

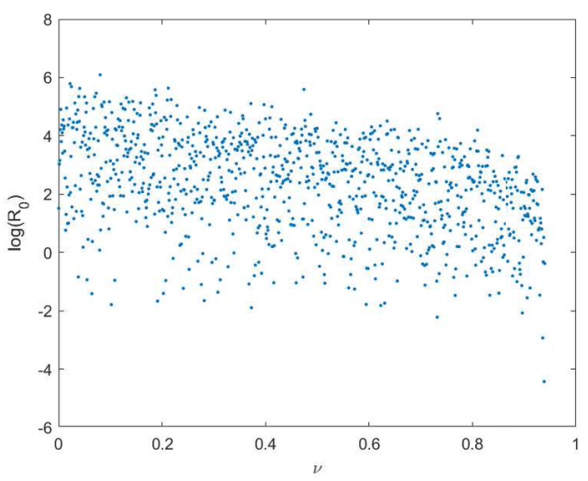

(b)

Fig. 2 The Monte Carlo simulations for some of the parameters with the greatest PRCC magnitudes, using values in Table 3 and 1000 simulations per run. The parameters are: a sporozoite invasion rate $\beta_{s}$ and $\mathbf{b}$ efficacy of pre-erythrocytic vaccine $v$

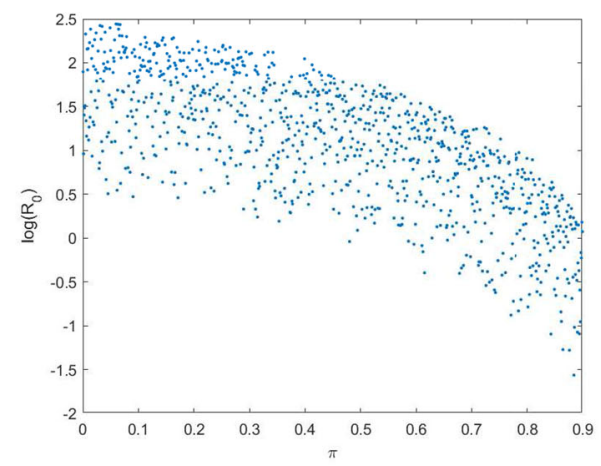

(a)

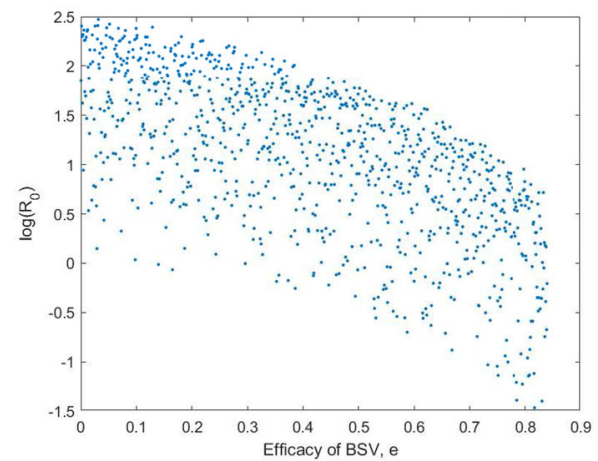

(c)

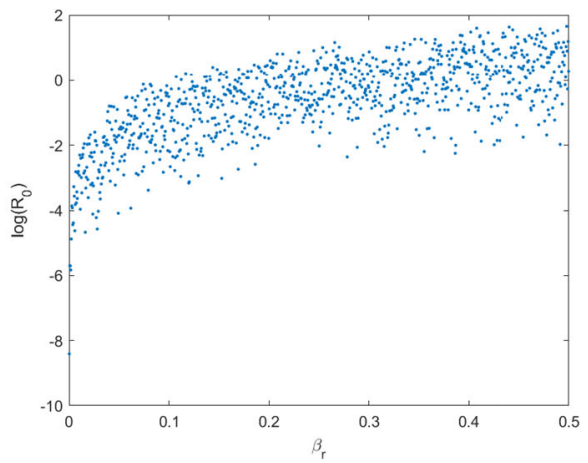

(b)

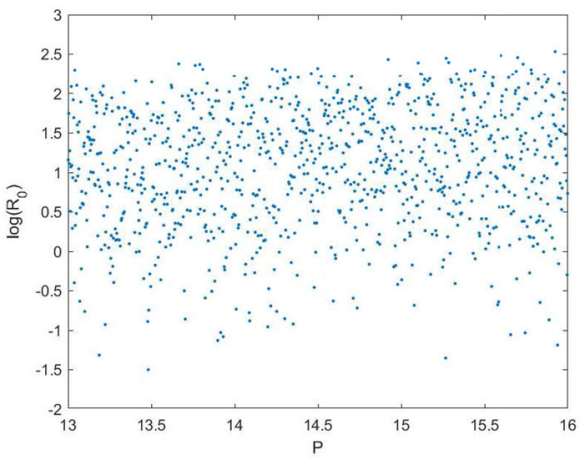

(d)

Fig. 3 The Monte Carlo simulations for some of the parameters with the greatest PRCC magnitudes, using values in Table 3 and 1000 simulations per run. They are: a proportion of parasites that become gametocytes $\pi, \mathbf{b}$ merozoite invasion rate $\beta_{r}$, c efficacy of blood stage vaccine $e$ and $\mathbf{d}$ the number of merozoites produced per bursting blood schizont $P$ 
Table 4 The sensitivity results

\begin{tabular}{llll}
\hline Parameter & S.I & Parameter & S.I \\
\hline$P$ & +1 & $a$ & -0.785714 \\
$\beta_{S}$ & +1 & $\pi$ & -0.785714 \\
$\lambda_{h}$ & +1 & $\beta_{r}$ & +0.62402 \\
$\mu_{h}$ & -1 & $\lambda_{r}$ & +0.62402 \\
$\mu_{x}$ & -1 & $\mu_{m}$ & -0.62406 \\
$\varrho$ & -0.785714 & $\mu_{r}$ & -0.62406 \\
$\nu$ & -0.785714 & $q$ & -0.428571 \\
\hline
\end{tabular}

and in the development of malaria vaccines. In response, we consider the normalized forward sensitivity index of the model reproduction number in (15) and (16) against LHS parameters in Table 3 in the next section.

\section{Sensitivity Analysis Based on Model $R_{0}$}

We adopt the normalized forward sensitivity (NFS) index technique described in [4] to compute the sensitivity indices of the model $R_{0}$ with respect to input parameters $P, \beta_{r}, \beta_{s}, \lambda_{h}, \lambda_{r}, \mu_{h}, \mu_{r}, \mu_{x}, \mu_{m}, \varrho, a, \pi, v$ are presented in Table 4 . The sensitivity indices (S.I) are evaluated in Mathematica software based on the baseline parameter values in Table 3.

From Table 4, we observe that the population of malaria merozoites that are released per bursting infected erythrocyte $P$, the rates of invasions of healthy erythrocytes $\beta_{r}$ and hepatocytes $\beta_{s}$, the rate of recruitment of susceptible hepatocytes $\lambda_{h}$ and erythrocytes $\lambda_{r}$ have a direct effects on the severity of clinical malaria. A $10 \%$ increase (or decrease) in the magnitude of $P$, increase (or decrease) model $R_{0}$ by $10 \%$. Clinical malaria control should aim at eradicating or reducing these parameters. On the other hand, malaria vaccines ( $\varrho$ and $v$ ) are shown to reduce $R_{0}$ when their efficacies are improved. An increase (or decrease) in the immunosensitivity of infected red blood cells $q$ is similarly shown to reduce (or increase) the model $R_{0}$. By rank, the parameters $P, \beta_{s}, \lambda_{h}$ are the most influential in model (1), followed by the vaccine efficacies $\varrho$ and $\nu$. Clinical considerations should thus favour these parameters in the development of malaria vaccines.

In conclusion, the LHS/PRCC technique is robust and considers the entire parameter space in determining significant parameters in a disease model. However, it fails the ranking test. On the other hand, although the NFS index technique is beneficial in discerning and ranking significant parameters in a model, it does not explain the considerable uncertainties in estimating parameter values. It therefore fails to uncover parameter effects on particular variables or populations in the model. Due to the importance attached to the significance and rank of each parameter in the model, we advise that both approaches be used in future analysis of parameter uncertainty and sensitivity analysis in disease models.

\section{Numerical Simulation}

In this section, we illustrate numerically the dynamics of the in-host model system (1). When the basic reproduction number $R_{0}$ is less than unity, clinical $P$. falciparum malaria is observed to die out (see Figs. 4,5$)$ and the malaria-free state $E_{0}=\left(0,5 \times 10^{5}, 0,0,5 \times\right.$ 


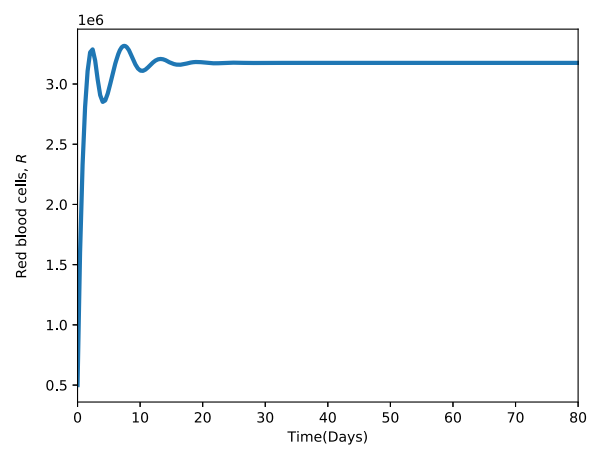

(a)

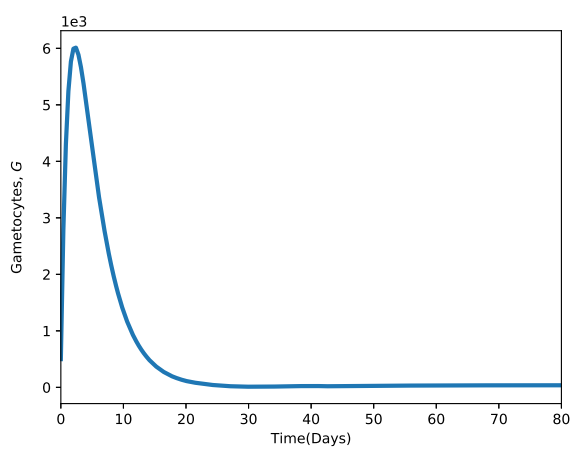

(c)

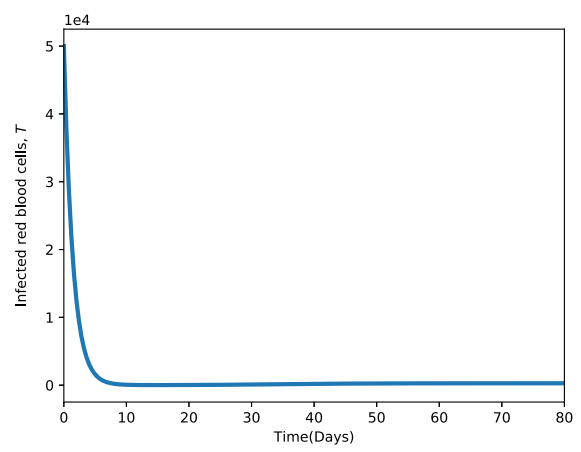

(b)

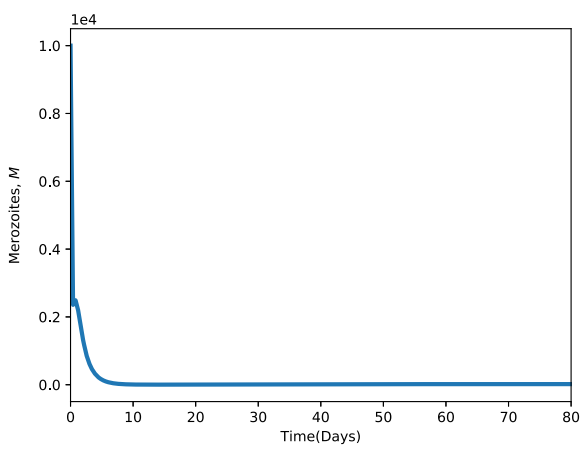

(d)

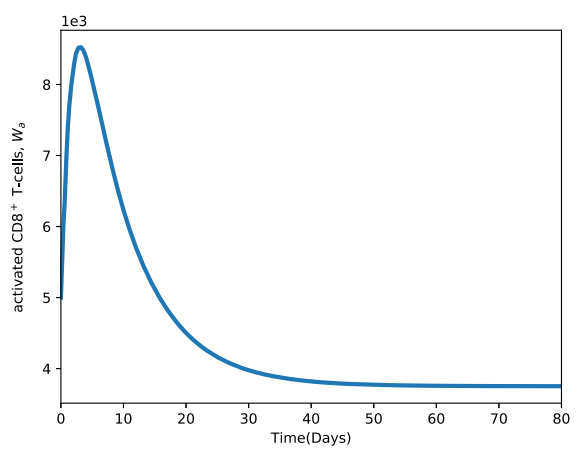

(e)

Fig. 4 The dynamics of model (1) when the basic reproduction number $R_{0}=0.8881<1$, the malaria-free equilibrium $E_{0}$ is globally asymptotically stable

$\left.10^{6}, 0,0,0,0,0\right)$ is globally asymptotically stable. Additionally, only an efficacious BSV has the chance to totally eradicate the merozoites from the blood stream of the human host. Notice that even if $R_{0}$ is less than unity, some activated CD8 ${ }^{+}$T-cells $W_{a}(t)$ still exists (see Fig. 4e). This shows that individual-level acquired immunity against malaria is maintained for some period of time even if all malaria parasites are eradicated from the host. All simulations are performed using parameter values in Table 3. 


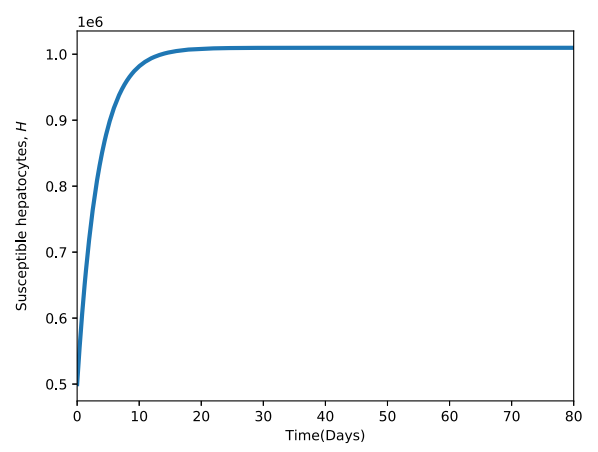

(a)

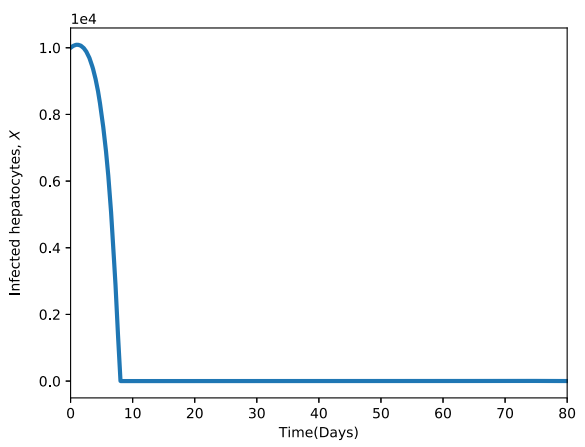

(b)

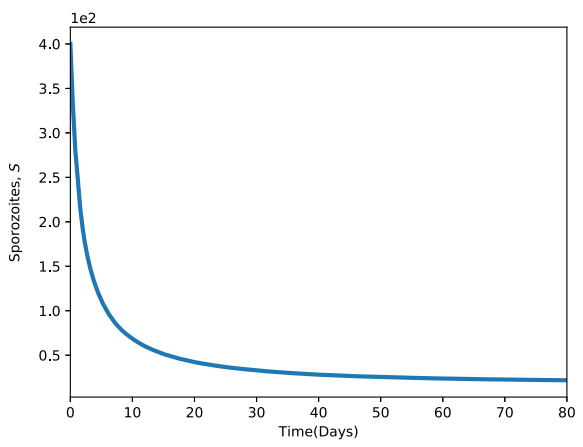

(c)

Fig. 5 Polulation dynamics of the liver hepatocytes and the sporozoites when the reproduction number $R_{0}=$ $0.8881<1$. Parameter values are available in Table 3

Similarly, the density of sporozoites decline rapidly when $R_{0}=R_{01}<1$, leading to a corresponding decrease in the density of infected hepatocytes as shown in Fig. 5.

The persistent steady state, $E_{p}=\left(S^{*}, H^{*}, X^{*}, R^{*}, T^{*}, M^{*}, G^{*}, W_{n}^{*}, W_{a}^{*}\right)=\left(10^{3}, 3 \times\right.$ $\left.10^{5}, 3 \times 10^{3}, 5 \times 10^{6}, 5 \times 10^{3}, 10^{3}, 5 \times 10^{2}, 5 \times 10^{3}, 5 \times 10^{3}\right)$ is shown to be stable when $R_{0}$ is greater than unity, $R_{0}=1.3364>1$ (See Figs. 6, 7). We observe that the densities of sporozoites, merozoites, gametocytes, infected hepatocytes and infected erythrocytes grow and persists both at the pre-erythrocytic and blood stages of parasite development.

\section{Multi-component and Multi-stage Vaccine Cocktail}

Here, we illustrate the need to develop a multi-component and multi-stage $P$. falciparum malaria vaccines with sufficient prospect to minimize the severity of malaria infection from human host. Several strategies have been considered in the development of efficient multi-component and multi-stage $P$. falciparum vaccine formulations $[16,51,53,57,58,67]$. A synthetic peptide (SPf66) that combines antigens from the blood stages of malaria linked together with an antigen from the sporozoite stage reduced the number of first attacks with $P$. falciparum by $28 \%$ in South America [24]. High level of sterile efficacy and safety of combining pre-erythrocytic vaccine, RTS,S/AS01B with vectored vaccines are reported in [53]. 


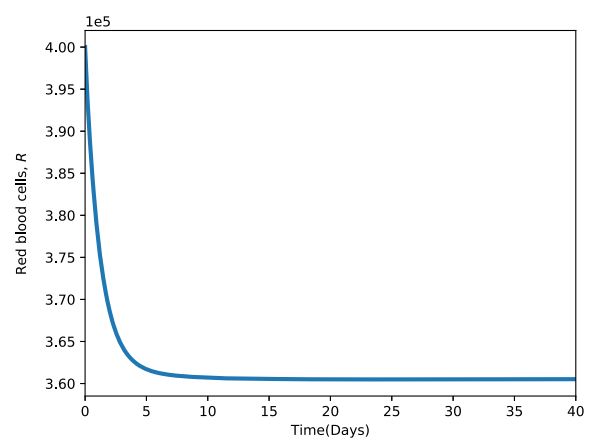

(a)

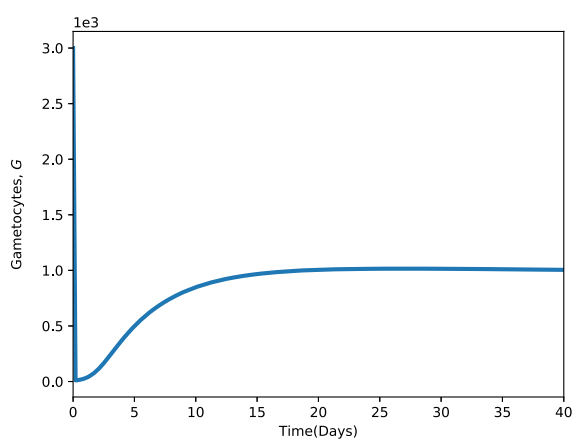

(c)

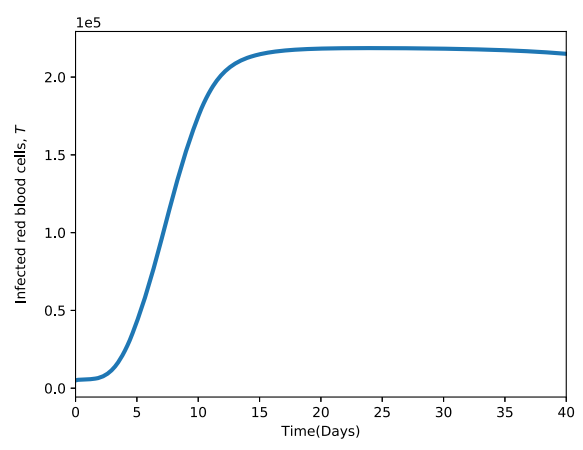

(b)

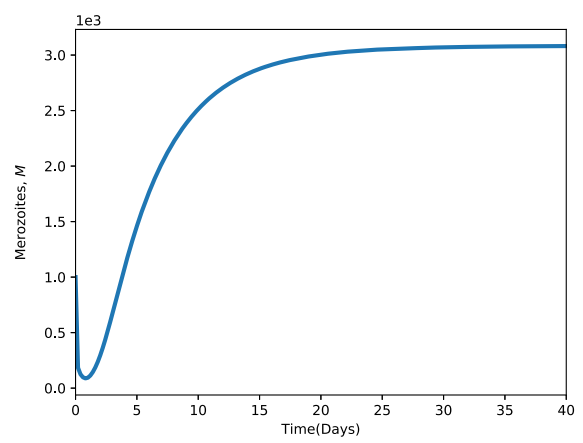

(d)

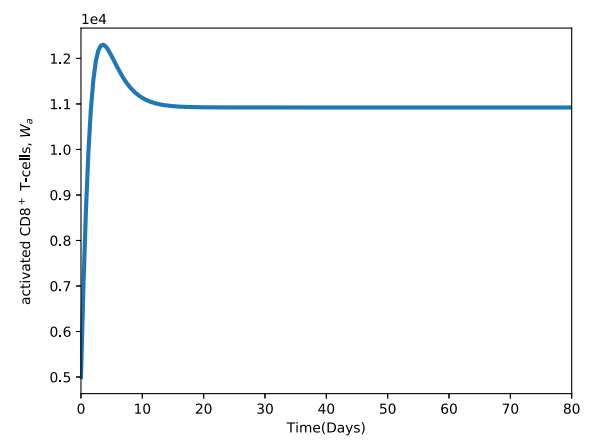

(e)

Fig. 6 When the reproduction number $R_{0}=1.3364>1$, the malaria-persistent equilibrium point $E_{p}$ is stable for the chosen set of parameter values. The model parameter values are available in Table 3

Plasmodium falciparum chimeric protein 2.9 which is a combination of the C-terminal regions of domain III of AMA-1 and 19-kDa of MSP1 induced both anti-MSP1-19 and antiAMA-1(III) Abs at levels 11- and 18-fold higher, respectively, than individual components did [51]. Studies in [19] have also shown that a combination of multiple vaccine candidates in fusion proteins may lead to improved characteristics of the vaccine. 


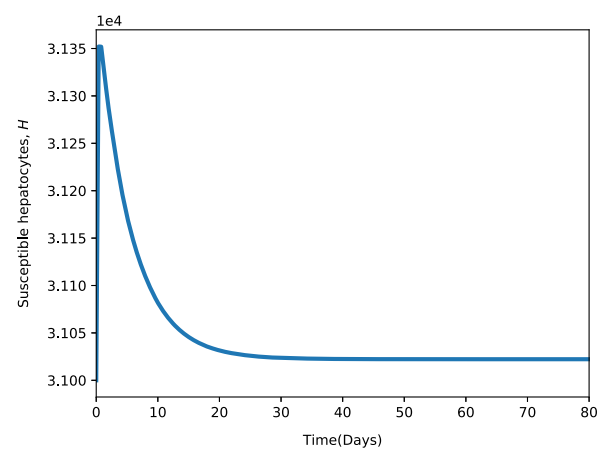

(a)

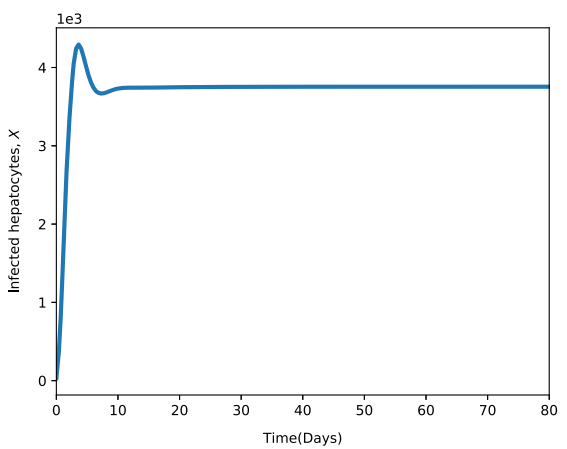

(b)

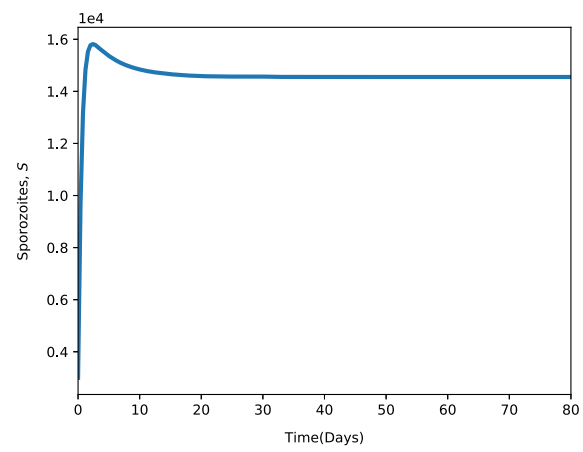

(c)

Fig. 7 When the reproduction number $R_{0}=1.3364>1$, the malaria-persistent equilibrium $E_{p}$ is stable

Synergism from combination of transmission blocking vaccines is shown in [67] to confer protection by simultaneously inducing multiple, independent immune responses directed towards $P$. falciparum sporozoites. This ensures high levels of vaccine efficacy against the parasite. The need for multi-stage malaria vaccine is also emphasised in [16] where DNA vaccines are shown to have high capacity to induce CD8(+) cytotoxic T lymphocytes and interferon-gamma responses in humans during $P$. falciparum infection [62].

From these studies, it is evident that a multi-stage specific vaccine cocktail has the potential to prevent initial malaria infection at the pre-erythrocytic stage, reduce or eradicate clinical manifestation and prevent the transmission of gametocytes from infected human hosts to susceptible feeding female anopheles mosquito [10]. We therefore evaluate numerically the effects of malaria vaccine combinations on the severity of $P$. falciparum malaria infection. The vaccines are broadly categorized as: pre-erythrocytic vaccines, blood stage vaccines and transmission blocking vaccines. The vaccines are assumed to have an optimal efficacy of $75 \%$. We chose this figure in consistency with the WHO strategic goal of developing a $P$. falciparum vaccine with atleast $75 \%$ efficacy [43].

Results in Fig. 8, shows the effects of all the three vaccines combined with a constant optimal efficacy of $75 \%$. The density of infected erythrocytes $T$ and infected hepatocytes $X$ is shown to fall significantly. The severity of malaria infection is therefore highly reduced when the three vaccine antigens are combined. 


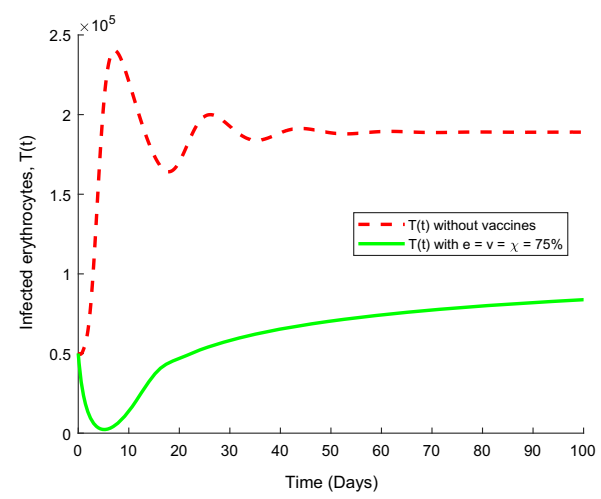

(a)

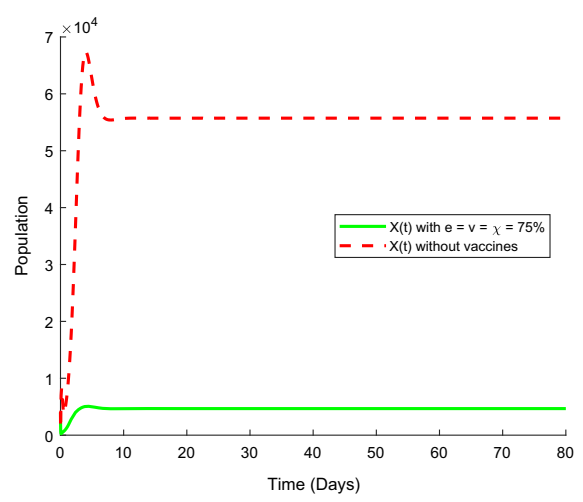

(b)

Fig. 8 Impact of a 75\% efficacious combined malaria vaccines on the dynamics of infected red blood cells $T$ and infected hepatocytes $X$. The simulations are performed when $R_{01}=2.564>1$, and $R_{02}=5.564>1$

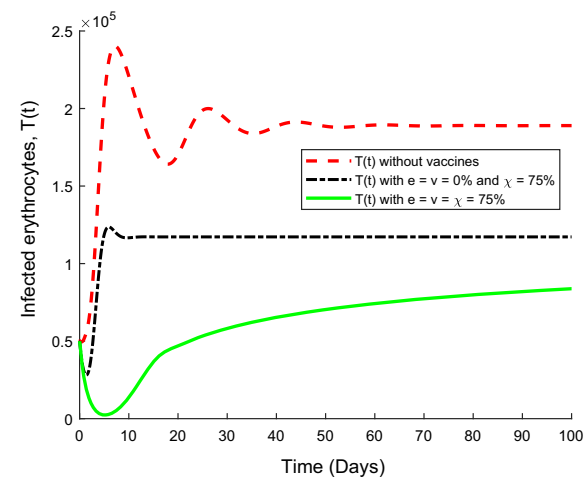

(a)

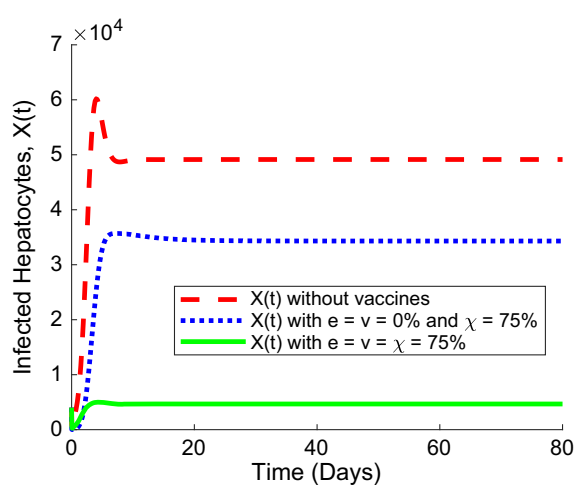

(b)

Fig. 9 Impact of a 75\% efficacious transmission blocking vaccine on the dynamics of infected red blood cells and infected hepatocytes. The simulations are performed when $R_{01}=2.564>1$, and $R_{02}=5.564>1$

The use of only one vaccine is shown in Figs. 9, 10 and 11 to have minimal effects on the severity of malaria infection.

Finally, a combination of any two vaccines (with different antigens) is likely to moderately reduce the severity of malaria infection. The effects of paired-vaccine combinations are as shown in Figs. 12, 13 and 14. Unlike infected hepatocytes, transmission blocking vaccines are shown to have minimal effects on the density of infected red blood cells (see Fig. 10).

A combination of an efficacious pre-erythrocytic vaccine and an effective blood stage vaccine antigens has the potential to significantly reduce malaria severity as shown in Fig. 14. Moreover, a combination of antigens from transmission blocking vaccine and those of preerythrocytic vaccine at the liver stage, as shown in Fig. 12b, are very effective in suppressing the density of infected hepatocytes in the liver. 


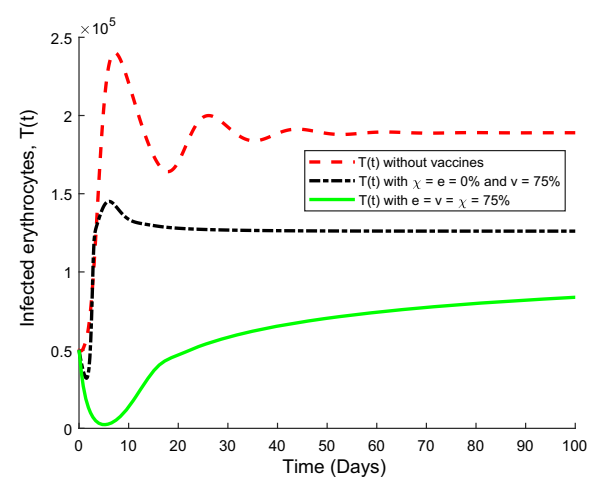

(a)

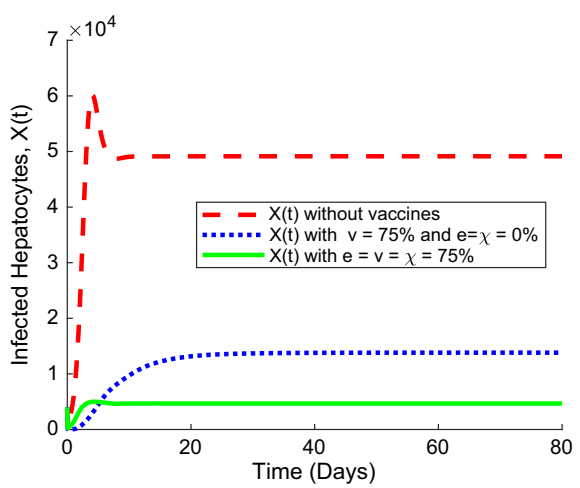

(b)

Fig. 10 Impact of a 75\% efficacious pre-erythrocytic vaccine on the dynamics of infected red blood cells and infected hepatocytes. The simulations are performed when $R_{01}=2.564>1$, and $R_{02}=5.564>1$

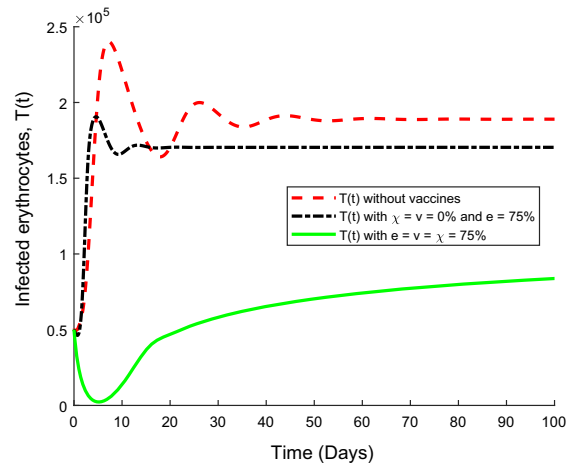

(a)

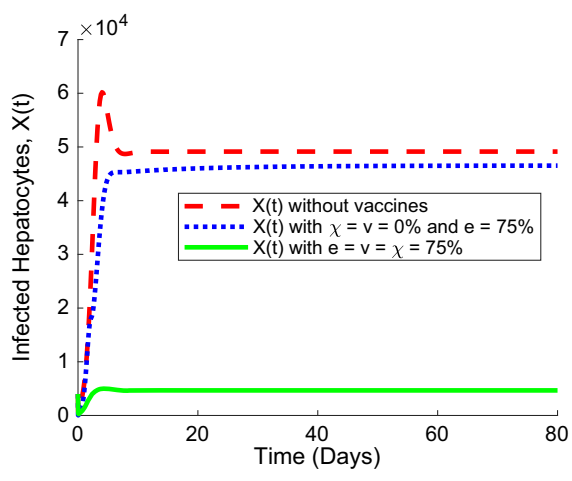

(b)

Fig. 11 Impact of a 75\% efficacious blood stage vaccine on the dynamics of infected red blood cells and infected hepatocytes. The simulations are performed when $R_{01}=2.564>1$, and $R_{02}=5.564>1$

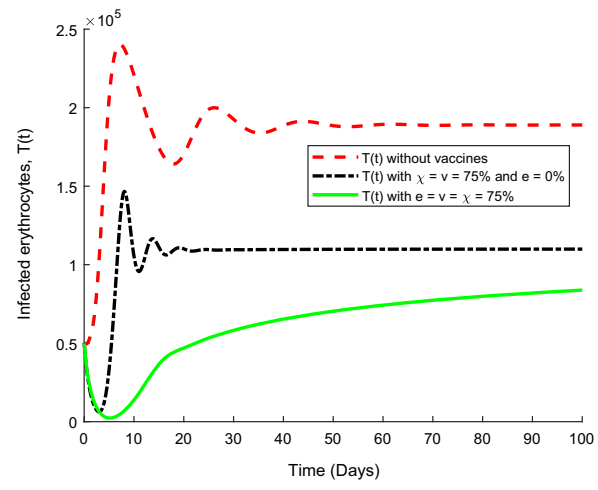

(a)

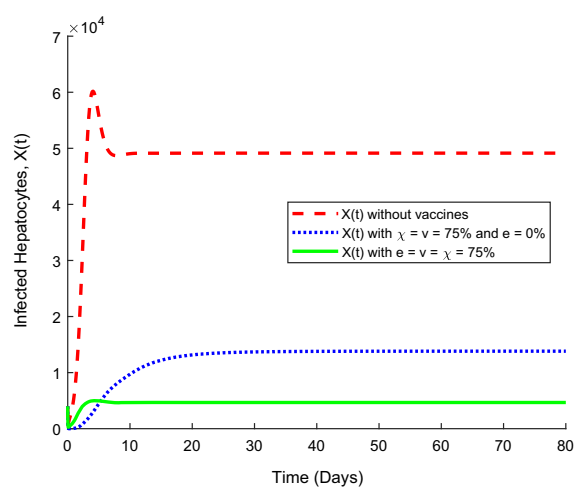

(b)

Fig. 12 The effect of combining a PEV and TBV that are both $75 \%$ efficacious on the dynamics of infected red blood cells and infected hepatocytes. The simulations are performed when $R_{01}=2.564>1$, and $R_{02}=5.564>1$ 


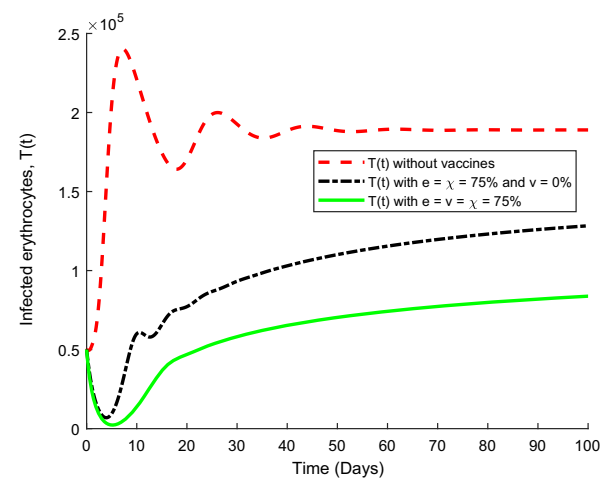

(a)

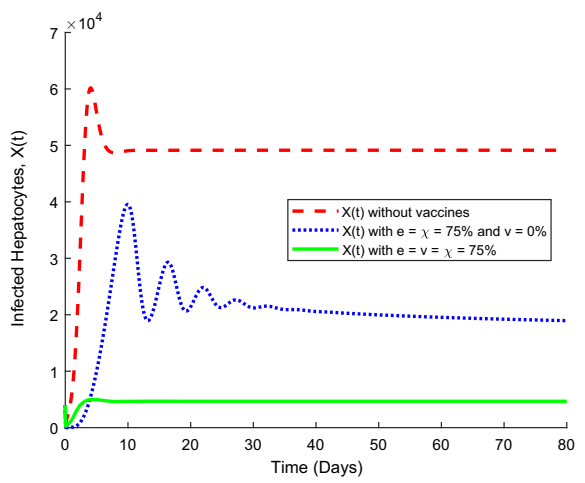

(b)

Fig. 13 The effect of combining a TBV and a PEV that are both $75 \%$ efficacious on the dynamics of infected red blood cells and infected hepatocytes. The simulations are performed when $R_{01}=2.564>1$, and $R_{02}=5.564>1$

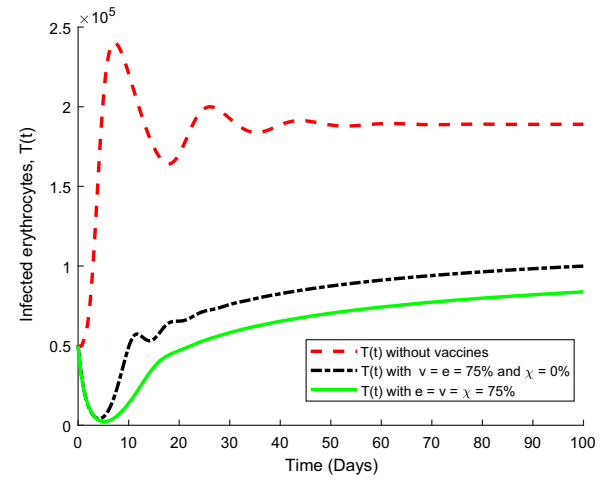

(a)

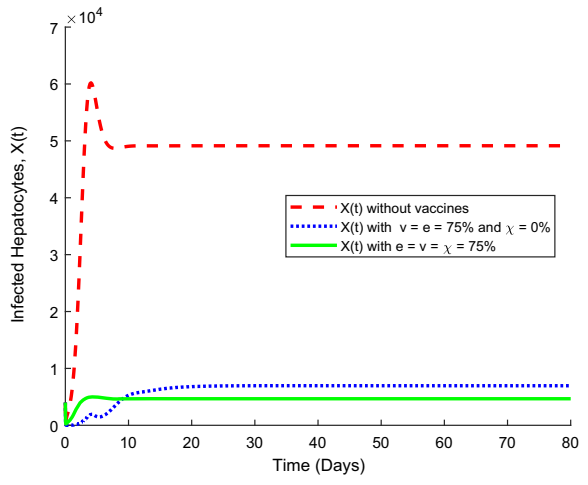

(b)

Fig. 14 The effect of combining a PEV and a BSV that are both $75 \%$ efficacious on the dynamics of infected red blood cells and infected hepatocytes. The simulations are performed when $R_{01}=2.564>1$, and $R_{02}=5.564>1$

\section{Epidemiological Implications and Conclusion}

Results of uncertainty and sensitivity analysis have significant epidemiological importance in malaria control. The presented model is shown to be sensitive to variations in vaccine efficacies $\chi, \varrho$ and $\nu$. Only efficacious malaria vaccines have a chance to eradicate $P$. falciparum malaria or to reduce its severity, significantly, during clinical infection. Sensitivity indices based on the model reproduction number indicates that the rate of invasion of susceptible erythrocytes by merozoite parasites $\beta_{r}$ and the rate of invasion of liver hepatocytes by the sporozoites $\beta_{s}$ are highly sensitive to malaria disease progression. Malaria vaccines must therefore target and eradicate the infective parasites and infected cells. Other parameters such as the proportions of merozoites that become gametocytes per dying blood schizont $\pi$ and the average number of merozoites released per bursting blood schizonts $P$ are also shown to have a considerable impact on the model $R_{0}$ and hence the severity of infection. 
A highly efficacious blood stage vaccine is necessary to lower the rate $\pi$. A reduction in the density of blood gametocytes has the potential of lowering malaria transmission from the human host to the mosquito vector.

Given the protective capacity of liver stage-specific $\mathrm{CD}^{+} \mathrm{T}$-cells [65], a lot more research that focuses on developing an efficacious blood stage vaccine that would prime blood trophozoite-specific $\mathrm{CD} 8^{+} \mathrm{T}$-cells is called for. The parameter $\tau$ which accounts for vaccineinduced production of $\mathrm{CD}^{+}$T-cells is shown to have significant negative effects on the concentration of infected hepatocytes. The more efficient the pre-erythrocytic vaccine is, the lower the density of released merozoites into the host's blood. Moreover, a rapid eradication of parasitized liver hepatocytes is likely to result into less infected erythrocytes at the blood stage. An efficacious pre-erythrocytic vaccines has a potential to eradicate infected liver hepatocytes. Such efficacious liver stage vaccines may also reduce the overall burst size of an infected hepatocyte, leading to reduced erythrocytic schizonogy and hence the severity of clinical $P$. falciparum malaria.

Multiple combination of malaria vaccine antigen has a great potential in reducing the severity of malaria infections due to $P$. falciparum parasite. The synergy of combining preerythrocytic vaccines, blood stage vaccines and transmission blocking vaccines antigens induces multiple immune responses with the potential to prevent or eradicate malaria infection. As highlighted in [32], our study shows that a highly effective vaccine combination is critical for $P$. falciparum malaria disease elimination goal.

Although efficacious malaria vaccines are shown to be very effective in reducing severity of clinical malaria, our results further confirm the need to combine vaccine with existing antimalarial therapy to achieve a complete eradication of the parasites from the host. Finally, the LHS/PRCC analysis revealed that the prediction imprecision was mainly due to certain key parameters. Long term precise predictions of the concentrations of infected liver hepatocytes and infected red blood cells would be difficult until these key parameters are correctly determined. A great understanding of parameter uncertainty and sensitivity analysis of in-host malaria in humans is necessary in order to develop effective vaccines against $P$. falciparum pathogen.

Acknowledgements The authors would like to thank the anonymous referees for their constructive comments. The authors also acknowledge with gratitude the support from the institute of Mathematical SciencesStrathmore university.

Author contributions All authors contributed to all sections of this manuscript.

Funding The authors acknowledge with gratitude the financial support from the German Academic Exchange Service (DAAD) [ST32-PKZ:91711149] and the national Research Fund (NRF)-Kenya [NRF-Phd Grant Titus $\mathrm{O} . \mathrm{O}]$, in the production of this manuscript.

\section{Compliance with Ethical Standards}

Conflict of interest The authors declare that there is no conflict of interest regarding the publication of this article.

\section{Appendix A: PRCC Between Infected Red Blood Cells $T$ and Each Param- eter}

See Table 5 . 
Table 5 PRCC between infected red blood cells $T$ and each parameter

\begin{tabular}{llllll}
\hline Parameter & PRCC & $p$ value & Parameter & PRCC & $p$ value \\
\hline$\Lambda$ & 0.040124 & 0.69185 & $v$ & $-0.35423^{*}$ & 0.0001255 \\
$\mu_{s}$ & 0.092654 & 0.35921 & $d$ & -0.021968 & 0.82825 \\
$\lambda_{h}$ & 0.16815 & 0.094459 & $\lambda_{w}$ & 0.02639 & 0.79438 \\
$\lambda_{r}$ & $0.99993^{* * *}$ & $1.2467 \mathrm{E}-191$ & $\tau$ & 0.028815 & 0.77596 \\
$\beta_{r}$ & $0.60394^{* *}$ & 0.0030343 & $a$ & -0.033076 & 0.7439 \\
$\beta_{S}$ & -0.072861 & 0.47128 & $b$ & -0.037844 & 0.70854 \\
$\mu_{h}$ & 0.045043 & 0.65633 & $P$ & $0.28652^{*}$ & 0.0038515 \\
$\mu_{x}$ & 0.022164 & 0.82674 & $N$ & $0.50348^{* *}$ & 0.0003056 \\
$\pi$ & 0.24376 & 0.14528 & $\epsilon_{0}$ & -0.050167 & 0.62012 \\
$\mu_{r}$ & $-0.54174^{* *}$ & $5.8708 \mathrm{E}-9$ & $\epsilon_{1}$ & 0.0092028 & 0.92759 \\
$\mu_{t}$ & -0.043585 & 0.66677 & $\rho$ & 0.087162 & 0.38852 \\
$\mu_{m}$ & -0.08574 & 0.39634 & $q$ & $-0.261905^{*}$ & 0.00154063 \\
$\mu_{g}$ & -0.13006 & 0.19715 & $\mu_{n}$ & -0.093091 & 0.35694 \\
$\chi$ & -0.13607 & 0.17706 & $\mu_{a}$ & -0.054864 & 0.58771 \\
$\varrho$ & -0.30701 & 0.0018909 & $\Omega$ & 0.041721 & 0.68024 \\
\hline
\end{tabular}

The results are significant at the 0.05 level

\section{Appendix B: PRCC Between Infected Hepatocytes $X$ and Each Parameter}

See Table 6.

Table 6 PRCC between infected hepatocytes $X$ and each parameter

\begin{tabular}{lcllll}
\hline Parameter & PRCC & $p$ value & Parameter & PRCC & $p$ value \\
\hline$\Lambda$ & -0.034437 & 0.73375 & $v$ & $-0.307831^{*}$ & 0.00093836 \\
$\mu_{s}$ & 0.071002 & 0.48269 & $d$ & -0.1442 & 0.15231 \\
$\lambda_{h}$ & $0.9749^{* * *}$ & $9.5419 \mathrm{E}-66$ & $\lambda_{w}$ & -0.094174 & 0.35135 \\
$\lambda_{r}$ & 0.06407 & 0.52654 & $\tau$ & $-0.20137^{*}$ & 0.000044531 \\
$\beta_{r}$ & -0.080809 & 0.42415 & $a$ & -0.075135 & 0.45751 \\
$\beta_{S}$ & $0.53926^{* *}$ & 0.00016701 & $b$ & 0.061933 & 0.54045 \\
$\mu_{h}$ & $-0.87273^{* * *}$ & $2.7908 \mathrm{E}-23$ & $P$ & 0.076347 & 0.45026 \\
$\mu_{x}$ & 0.14762 & 0.14273 & $N$ & 0.10516 & 0.29776 \\
$\pi$ & -0.045846 & 0.6506 & $\epsilon_{0}$ & 0.21125 & 0.034877 \\
$\mu_{r}$ & 0.094998 & 0.34713 & $\epsilon_{1}$ & -0.16583 & 0.09918 \\
$\mu_{t}$ & 0.12588 & 0.21205 & $\rho$ & 0.033235 & 0.74272 \\
$\mu_{m}$ & 0.13493 & 0.18073 & $q$ & 0.095106 & 0.34658 \\
$\mu_{g}$ & 0.033064 & 0.74399 & $\mu_{n}$ & 0.039223 & 0.69843 \\
$\chi$ & -0.28902 & 0.0035417 & $\mu_{a}$ & -0.052237 & 0.60575 \\
$\varrho$ & -0.061742 & 0.5417 & $\Omega$ & 0.10411 & 0.30264 \\
\hline
\end{tabular}

The results are significant at the 0.05 level 


\section{Appendix C: PRCC Between Merozoites $M$ and Each Parameter}

See Table 7.

Table 7 PRCC between merozoites $M$ and each parameter

\begin{tabular}{lcllll}
\hline Parameter & PRCC & $p$ value & Parameter & PRCC & $p$ value \\
\hline$\Lambda$ & 0.013086 & 0.89718 & $v$ & $-0.61866^{* *}$ & $6.9194 \mathrm{E}-12$ \\
$\mu_{s}$ & 0.12012 & 0.23389 & $d$ & 0.12239 & 0.22509 \\
$\lambda_{h}$ & -0.005884 & 0.95367 & $\lambda_{w}$ & -0.30208 & 0.0022545 \\
$\lambda_{r}$ & $-0.78852^{* *}$ & $2.0731 \mathrm{E}-22$ & $\tau$ & -0.11155 & 0.26918 \\
$\beta_{r}$ & $-0.77199^{* *}$ & $5.3565 \mathrm{E}-21$ & $a$ & 0.14416 & 0.15243 \\
$\beta_{s}$ & 0.10834 & 0.28328 & $b$ & -0.18907 & 0.059575 \\
$\mu_{h}$ & 0.080773 & 0.42436 & $P$ & $0.28652^{*}$ & 0.0038515 \\
$\mu_{x}$ & $0.46617^{*}$ & $1.0183 \mathrm{E}-06$ & $N$ & $-0.50348^{* *}$ & 0.0003056 \\
$\pi$ & -0.29849 & 0.0025577 & $\epsilon_{0}$ & -0.23892 & 0.01667 \\
$\mu_{r}$ & 0.060227 & 0.55168 & $\epsilon_{1}$ & -0.057548 & 0.56955 \\
$\mu_{t}$ & -0.05975 & 0.55485 & $\rho$ & -0.25708 & 0.0098207 \\
$\mu_{m}$ & -0.090522 & 0.37042 & $q$ & -0.047855 & 0.63635 \\
$\mu_{g}$ & 0.18256 & 0.069072 & $\mu_{n}$ & -0.0015737 & 0.9876 \\
$\chi$ & $-0.67251^{* *}$ & $1.8298 \mathrm{E}-14$ & $\mu_{a}$ & 0.1363 & 0.17632 \\
$\varrho$ & -0.024582 & 0.80818 & $\Omega$ & -0.12172 & 0.22769 \\
\hline
\end{tabular}

The results are significant at the 0.05 level

\section{Appendix D: PRCC Between Gametocytes G and Each Parameter}

See Table 8 . 
Table 8 PRCC between gametocytes $G$ and each parameter

\begin{tabular}{llllll}
\hline Parameter & PRCC & $p$ value & Parameter & PRCC & $p$ value \\
\hline$\Lambda$ & 0.09861 & 0.32902 & $v$ & $-0.54919^{* *}$ & $3.2858 \mathrm{E}-09$ \\
$\mu_{s}$ & -0.18356 & 0.067537 & $d$ & -0.33627 & 0.00062476 \\
$\lambda_{h}$ & 0.16929 & 0.092216 & $\lambda_{w}$ & -0.14215 & 0.15831 \\
$\lambda_{r}$ & $-0.79454^{* * *}$ & $5.8878 \mathrm{E}-23$ & $\tau$ & -0.080527 & 0.42578 \\
$\beta_{r}$ & $-0.76958^{* *}$ & $8.4228 \mathrm{E}-21$ & $a$ & -0.16499 & 0.10092 \\
$\beta_{S}$ & -0.021251 & 0.83378 & $b$ & -0.31287 & 0.0015279 \\
$\mu_{h}$ & 0.060337 & 0.55095 & $P$ & 0.059063 & 0.55941 \\
$\mu_{x}$ & $0.40198^{*}$ & $3.3899 \mathrm{E}-05$ & $N$ & 0.24601 & 0.013619 \\
$\pi$ & $0.698883^{* *}$ & 0.0032768 & $\epsilon_{0}$ & -0.28727 & 0.0037563 \\
$\mu_{r}$ & -0.022582 & 0.82353 & $\epsilon_{1}$ & 0.075806 & 0.45349 \\
$\mu_{t}$ & 0.075502 & 0.45531 & $\rho$ & -0.047433 & 0.63933 \\
$\mu_{m}$ & 0.067991 & 0.50149 & $q$ & 0.12264 & 0.22416 \\
$\mu_{g}$ & -0.084857 & 0.40124 & $\mu_{n}$ & -0.12368 & 0.22021 \\
$\chi$ & $-0.55186^{* *}$ & $2.6601 \mathrm{E}-09$ & $\mu_{a}$ & 0.17443 & 0.082611 \\
$\varrho$ & $-0.55799^{* *}$ & $1.6273 \mathrm{E}-09$ & $\Omega$ & 0.15261 & 0.12956 \\
\hline
\end{tabular}

The results are significant at the 0.05 level

\section{Appendix E: PRCC Between Sporozoites S and Each Parameter}

See Table 9.

Table 9 PRCC between sporozoites $S$ and each parameter

\begin{tabular}{lcllll}
\hline Parameter & PRCC & $p$ value & Parameter & PRCC & $p$ value \\
\hline$\Lambda$ & $0.31314^{*}$ & 0.00097533 & $v$ & 0.0067811 & 0.94661 \\
$\mu_{s}$ & $-0.68707^{* *}$ & 0.0094591 & $d$ & 0.010905 & 0.91425 \\
$\lambda_{h}$ & -0.012635 & 0.90071 & $\lambda_{w}$ & 0.0043436 & 0.96579 \\
$\lambda_{r}$ & 0.03593 & 0.72266 & $\tau$ & 0.023508 & 0.81642 \\
$\beta_{r}$ & 0.025432 & 0.80168 & $a$ & 0.0065303 & 0.94859 \\
$\beta_{s}$ & -0.0091069 & 0.92835 & $b$ & -0.017604 & 0.86199 \\
$\mu_{h}$ & 0.022838 & 0.82156 & $P$ & -0.019561 & 0.84683 \\
$\mu_{x}$ & 0.0056574 & 0.95545 & $N$ & -0.015923 & 0.87505 \\
$\pi$ & -0.0047473 & 0.96261 & $\epsilon_{0}$ & -0.00027845 & 0.99781 \\
$\mu_{r}$ & 0.00025243 & 0.99801 & $\epsilon_{1}$ & -0.0003386 & 0.99733 \\
$\mu_{t}$ & -0.011016 & 0.91338 & $\rho$ & -0.031317 & 0.75709 \\
$\mu_{m}$ & 0.0096995 & 0.9237 & $q$ & -0.0037326 & 0.9706 \\
$\mu_{g}$ & -0.023075 & 0.81974 & $\mu_{n}$ & 0.030253 & 0.7651 \\
$\chi$ & $-0.699368^{*}$ & 0.0001184 & $\mu_{a}$ & -0.0042258 & 0.96672 \\
$\varrho$ & 0.01863 & 0.85403 & $\Omega$ & -0.0052564 & 0.95861 \\
\hline
\end{tabular}

The results are significant at the 0.05 level 


\section{References}

1. Anderson, R., May, R., Gupta, S.: Non-linear phenomena in host-parasite interactions. Parasitology 99(S1), S59-S79 (1989)

2. Arama, C., Troye-Blomberg, M.: The path of malaria vaccine development: challenges and perspectives. J. Intern. Med. 275(5), 456-466 (2014)

3. Arevalo-Herrera, M., Solarte, Y., Yasnot, M.F., Castellanos, A., Rincon, A., Saul, A., Mu, J., Long, C., Miller, L., Herrera, S.: Induction of transmission-blocking immunity in Aotus monkeys by vaccination with a Plasmodium vivax clinical grade PVS25 recombinant protein. Am. J. Trop. Med. Hyg. 73(5-Suppl), 32-37 (2005)

4. Arriola, L., Hyman, J.: Lecture notes, forward and adjoint sensitivity analysis: with applications in dynamical systems. Linear Algebra and Optimisation Mathematical and Theoretical Biology Institute, Summer (2005)

5. Audran, R., Cachat, M., Lurati, F., Soe, S., Leroy, O., Corradin, G., Druilhe, P., Spertini, F.: Phase i malaria vaccine trial with a long synthetic peptide derived from the merozoite surface protein 3 antigen. Infect. Immun. 73(12), 8017-8026 (2005)

6. Birkett, A.J.: Status of vaccine research and development of vaccines for malaria. Vaccine 34(26), 29152920 (2016)

7. Birkett, A.J., Moorthy, V.S., Loucq, C., Chitnis, C.E., Kaslow, D.C.: Malaria vaccine R\&D in the decade of vaccines: breakthroughs, challenges and opportunities. Vaccine 31, B233-B243 (2013)

8. Blower, S.M., Dowlatabadi, H.: Sensitivity and uncertainty analysis of complex models of disease transmission: an HIV model, as an example. Int. Stat. Rev. 62(2), 229-243 (1994)

9. Blower, S.M., Hartel, D., Dowlatabadi, H., Anderson, R.M., May, R.M.: Drugs, sex and HIV: a mathematical model for New York city. Philos. Trans. R. Soc. Lond. B 331(1260), 171-187 (1991)

10. Boes, A., Spiegel, H., Voepel, N., Edgue, G., Beiss, V., Kapelski, S., Fendel, R., Scheuermayer, M., Pradel, G., Bolscher, J.M., et al.: Analysis of a multi-component multi-stage malaria vaccine candidate-tackling the cocktail challenge. PLoS ONE 10(7), e0131456 (2015)

11. Castillo-Chavez, C., Song, B.: Dynamical models of tuberculosis and their applications. Math. Biosci. Eng. 1(2), 361-404 (2004)

12. Cowman, A.F., Crabb, B.S.: Invasion of red blood cells by malaria parasites. Cell 124(4), 755-766 (2006)

13. Diebner, H.H., Eichner, M., Molineaux, L., Collins, W.E., Jeffery, G.M., Dietz, K.: Modelling the transition of asexual blood stages of Plasmodium falciparum to gametocytes. J. Theor. Biol. 202(2), 113-127 (2000)

14. Diekmann, O., Heesterbeek, J.A.P., Metz, J.A.: On the definition and the computation of the basic reproduction ratio $\mathrm{r} 0$ in models for infectious diseases in heterogeneous populations. J. Math. Biol. 28(4), 365-382 (1990)

15. Dondorp, A.M., Kager, P.A., Vreeken, J., White, N.J.: Abnormal blood flow and red blood cell deformability in severe malaria. Parasitol. Today 16(6), 228-232 (2000)

16. Doolan, D.L., Hoffman, S.L.: Dna-based vaccines against malaria: status and promise of the multi-stage malaria DNA vaccine operation. Int. J. Parasitol. 31(8), 753-762 (2001)

17. ECDC: Prevention and control measures for malaria (2018). http://ecdc.europa.eu/en/malaria/preventionand-control. Retrieved June 2018

18. EMA: First malaria vaccine receives positive scientific opinion from EMA. European Medicines Agency (2015). https://www.ema.europa.eu/en/news/first-malaria-vaccine-receives-positive-scientific-opinionema. Accessed March 2019

19. Faber, B.W., Younis, S., Remarque, E.J., Garcia, R.R., Riasat, V., Walraven, V., van der Werff, N., van der Eijk, M., Cavanagh, D.R., Holder, A.A., et al.: Diversity covering AMA1-MSP119 fusion proteins as malaria vaccines. Infect. Immun. pages IAI-01267 (2013)

20. Frischknecht, F., Baldacci, P., Martin, B., Zimmer, C., Thiberge, S., Olivo-Marin, J.-C., Shorte, S.L., Ménard, R.: Imaging movement of malaria parasites during transmission by anopheles mosquitoes. Cell. Microbiol. 6(7), 687-694 (2004)

21. Gomero, B.: Latin hypercube sampling and partial rank correlation coefficient analysis applied to an optimal control problem (2012)

22. Gomes, A.P., Vitorino, R.R., Costa, A.d P., Mendonça, E.G.d, Oliveira, M.G.d A., Siqueira-Batista, R.: Severe Plasmodium falciparum malaria. Rev. Bras. Terapia Intensiva 23(3), 358-369 (2011)

23. Gravenor, M., Kwiatkowski, D.: An analysis of the temperature effects of fever on the intra-host population dynamics of Plasmodium falciparum. Parasitology 117(2), 97-105 (1998)

24. Graves, P.M., Gelband, H.: Vaccines for preventing malaria (SPf66). Cochrane Database Syst. Rev. (2), CD005966 (2006). https://doi.org/10.1002/14651858.CD005966

25. Gurarie, D., McKenzie, F.E.: Dynamics of immune response and drug resistance in malaria infection. Malar. J. 5(1), 86-101 (2006) 
26. Hamby, D.: A review of techniques for parameter sensitivity analysis of environmental models. Environ. Monit. Assess. 32(2), 135-154 (1994)

27. Hetzel, C., Anderson, R.: The within-host cellular dynamics of bloodstage malaria: theoretical and experimental studies. Parasitology 113(1), 25-38 (1996)

28. Hisaeda, H., Stowers, A.W., Tsuboi, T., Collins, W.E., Sattabongkot, J.S., Suwanabun, N., Torii, M., Kaslow, D.C.: Antibodies to malaria vaccine candidates PVS25 and PVS28 completely block the ability of plasmodium vivax to infect mosquitoes. Infect. Immun. 68(12), 6618-6623 (2000)

29. Hoshen, M., Heinrich, R., Stein, W., Ginsburg, H.: Mathematical modelling of the within-host dynamics of Plasmodium falciparum. Parasitology 121(3), 227-235 (2000)

30. Iman, R.L., Helton, J.C.: An investigation of uncertainty and sensitivity analysis techniques for computer models. Risk Anal. 8(1), 71-90 (1988)

31. Inc., W.R. SystemModeler, Version 5.1. Champaign, IL (2018)

32. Kumar, N.: A vaccine to prevent transmission of human malaria: a long way to travel on a dusty and often bumpy road. Current Sci. 92, 1535-1544 (2007)

33. LaSalle, J.: The Stability of Dynamical Systems, Volume 25 of Regional Conference Series in Applied Mathematics. SIAM, Philadelphia (1976)

34. Li, M.Y., Muldowney, J.S.: Global stability for the seir model in epidemiology. Math. Biosci. 125(2), 155-164 (1995)

35. Li, M.Y., Shu, H.: Global dynamics of an in-host viral model with intracellular delay. Bull. Math. Biol. 72(6), 1492-1505 (2010)

36. Li, Y., Ruan, S., Xiao, D.: The within-host dynamics of malaria infection with immune response. Math. Biosci. Eng. 8(4), 999-1018 (2011)

37. Magombedze, G., Chiyaka, C., Mukandavire, Z.: Optimal control of malaria chemotherapy. Nonlinear Anal. Modell. Control 16(4), 415-434 (2011)

38. McKay, M.D., Beckman, R.J., Conover, W.J.: Comparison of three methods for selecting values of input variables in the analysis of output from a computer code. Technometrics 21(2), 239-245 (1979)

39. McQueen, P.G., McKenzie, F.E.: Age-structured red blood cell susceptibility and the dynamics of malaria infections. Proc. Natl. Acad. Sci. USA 101(24), 9161-9166 (2004)

40. Molineaux, L., Diebner, H., Eichner, M., Collins, W., Jeffery, G., Dietz, K.: Plasmodium falciparum parasitaemia described by a new mathematical model. Parasitology 122(4), 379-391 (2001)

41. Molineaux, L., Dietz, K.: Review of intra-host models of malaria. Parassitologia 41(1/3), 221-232 (1999)

42. Moorthy, V.S., Good, M.F., Hill, A.V.: Malaria vaccine developments. The Lancet 363(9403), 150-156 (2004)

43. MVFG: Malaria vaccine technology roadmap. Autoimmunity Research Foundation (2018). Retrieved from http://www.who.int/immunization/topics/malaria/vaccine_roadmap/en/. Accessed Aug 2018

44. Neilan, R.L.M., Schaefer, E., Gaff, H., Fister, K.R., Lenhart, S.: Modeling optimal intervention strategies for cholera. Bull. Math. Biol. 72(8), 2004-2018 (2010)

45. Niger, A.M., Gumel, A.B.: Immune response and imperfect vaccine in malaria dynamics. Math. Popul. Stud. 18(2), 55-86 (2011)

46. Nunes, J.K., Woods, C., Carter, T., Raphael, T., Morin, M.J., Diallo, D., Leboulleux, D., Jain, S., Loucq, C., Kaslow, D.C.: Development of a transmission-blocking malaria vaccine: progress, challenges, and the path forward. Vaccine 32(43), 5531-5539 (2014)

47. Ogutu, B.R., Apollo, O.J., McKinney, D., Okoth, W., Siangla, J., Dubovsky, F., Tucker, K., Waitumbi, J.N., Diggs, C., Wittes, J., et al.: Blood stage malaria vaccine eliciting high antigen-specific antibody concentrations confers no protection to young children in western Kenya. PLoS ONE 4(3), e4708 (2009)

48. Omondi, E.O., Orwa, T.O., Nyabadza, F.: Application of optimal control to the onchocerciasis transmission model with treatment. Math. Biosci. 297, 43-57 (2017)

49. Orwa, T.O., Mbogo, R.W., Luboobi, L.S.: Mathematical model for hepatocytic-erythrocytic dynamics of malaria. Int. J. Math. Math. Sci. 2018, 7019868 (2018). https://doi.org/10.1155/2018/7019868

50. Orwa, T.O., Mbogo, R.W., Luboobi, L.S.: Mathematical model for the in-host malaria dynamics subject to malaria vaccines. Lett. Biomath. 5(1), 222-251 (2018b)

51. Pan, W., Huang, D., Zhang, Q., Qu, L., Zhang, D., Zhang, X., Xue, X., Qian, F.: Fusion of two malaria vaccine candidate antigens enhances product yield, immunogenicity, and antibody-mediated inhibition of parasite growth in vitro. J. Immunol. 172(10), 6167-6174 (2004)

52. Pereira, A., Broed, R.: Methods for uncertainty and sensitivity analysis: review and recomendations for implementation in ecolego. Fysikum, Stockholm (2006). http://urn.kb.se/resolve?urn=urn:nbn:se:su: diva-1079. Accessed Oct 2018

53. Rampling, T., Ewer, K.J., Bowyer, G., Bliss, C.M., Edwards, N.J., Wright, D., Payne, R.O., Venkatraman, N., de Barra, E., Snudden, C.M., et al.: Safety and high level efficacy of the combination malaria vaccine 
regimen of RTS, S/AS01B with chimpanzee adenovirus 63 and modified vaccinia ankara vectored vaccines expressing me-trap. J. Infect. Dis. 214(5), 772-781 (2016)

54. Rts, S.C.T.P.: Efficacy and safety of the RTS, S/AS01 malaria vaccine during 18 months after vaccination: a phase 3 randomized, controlled trial in children and young infants at 11 African sites. PLoS Med. 11(7), e1001685 (2014)

55. Saul, A.: Models for the in-host dynamics of malaria revisited: errors in some basic models lead to large over-estimates of growth rates. Parasitology 117(5), 405-407 (1998)

56. Selemani, M.A., Luboobi, L.S., Nkansah-Gyekye, Y.: On stability of the in-human host and in-mosquito dynamics of malaria parasite. Asian J. Math. Appl. 2016, ama0353 (2016)

57. Stoute, J.A., Slaoui, M., Heppner, D.G., Momin, P., Kester, K.E., Desmons, P., Wellde, B.T., Garçon, N., Krzych, U., Marchand, M., et al.: A preliminary evaluation of a recombinant circumsporozoite protein vaccine against plasmodium falciparum malaria. New Engl. J. Med. 336(2), 86-91 (1997)

58. Stowers, A.W., Kennedy, M.C., Keegan, B.P., Saul, A., Long, C.A., Miller, L.H.: Vaccination of monkeys with recombinant Plasmodium falciparum apical membrane antigen 1 confers protection against bloodstage malaria. Infect. Immun. 70(12), 6961-6967 (2002)

59. Talman, A.M., Domarle, O., McKenzie, F.E., Ariey, F., Robert, V.: Gametocytogenesis: the puberty of Plasmodium falciparum. Malar. J. 3(1), 24 (2004)

60. Tumwiine, J., Hove-Musekwa, S.D., Nyabadza, F.: A mathematical model for the transmission and spread of drug sensitive and resistant malaria strains within a human population. ISRN Biomathematics (2014)

61. Tumwiine, J., Mugisha, J., Luboobi, L.: On global stability of the intra-host dynamics of malaria and the immune system. J. Math. Anal. Appl. 341(2), 855-869 (2008)

62. Tuteja, R.: DNA vaccine against malaria: a long way to go. Crit. Rev. Biochem. Mol. Biol. 37(1), 29-54 (2002)

63. Van den Driessche, P., Watmough, J.: Reproduction numbers and sub-threshold endemic equilibria for compartmental models of disease transmission. Math. Biosci. 180(1), 29-48 (2002)

64. Vaughan, A.M., Aly, A.S., Kappe, S.H.: Malaria parasite pre-erythrocytic stage infection: gliding and hiding. Cell Host Microbe 4(3), 209-218 (2008)

65. Villarino, N., W Schmidt, N.: Cd8+t cell responses to plasmodium and intracellular parasites. Curr. Immunol. Rev. 9(3), 169-178 (2013)

66. White, E., Comiskey, C.: Heroin epidemics, treatment and ODE modelling. Math. Biosci. 208(1), 312-324 (2007)

67. White, M.T., Smith, D.L.: Synergism from combinations of infection-blocking malaria vaccines. Malar. J. 12(1), 280 (2013)

68. WHO: Tables of malaria vaccine projects globally. "The Rainbow Tables" (2015). World Health Organization. https://www.who.int/immunization/research/development/Rainbow_tables/en/. Accessed Mar 2019

69. WHO: Overview of malaria treatment (2017). http://www.who.int/gho/malaria/areas/treatment/ overview/en/. Accessed Nov 2017

70. WHO: Malaria vaccine: WHO position paper, January 2016-recommendations. Vaccine 36(25), 35763577 (2018)

71. Zhong, P.: Optimal theory applied in integrodifference equation models and in a cholera differential equation model. The University of Tennessee Knoxville (2011). https://trace.tennessee.edu/cgi/viewcontent. cgi?referer=https://www.google.com/\&httpsredir=1\&article=2287\&context=utk_graddiss

Publisher's Note Springer Nature remains neutral with regard to jurisdictional claims in published maps and institutional affiliations. 